\title{
Spaces of phylogenetic networks from generalized nearest-neighbor interchange operations
}

\author{
Katharina T. Huber · Simone Linz · Vincent Moulton · \\ Taoyang Wu
}

Received: date / Accepted: date

\begin{abstract}
Phylogenetic networks are a generalization of evolutionary or phylogenetic trees that are used to represent the evolution of species which have undergone reticulate evolution. In this paper we consider spaces of such networks defined by some novel local operations that we introduce for converting one phylogenetic network into another. These operations are modeled on the well-studied nearest-neighbor interchange (NNI) operations on phylogenetic trees, and lead to natural generalizations of the tree spaces that have been previously associated to such operations. We present several results on spaces of some relatively simple networks, called level-1 networks, including the size of the neighborhood of a fixed network, and bounds on the diameter of the metric defined by taking the smallest number of operations required to convert one network into another. We expect that our results will be useful in the development of methods for systematically searching for optimal phylogenetic networks using, for example, likelihood and Bayesian approaches.
\end{abstract}

Keywords Phylogenetic networks - Spaces of phylogenetic trees $\cdot$ Phylogenetic tree metrics $\cdot$ Nearest-neighbor interchange (NNI)

Mathematics Subject Classification (2000) 05 C90 · 92D15

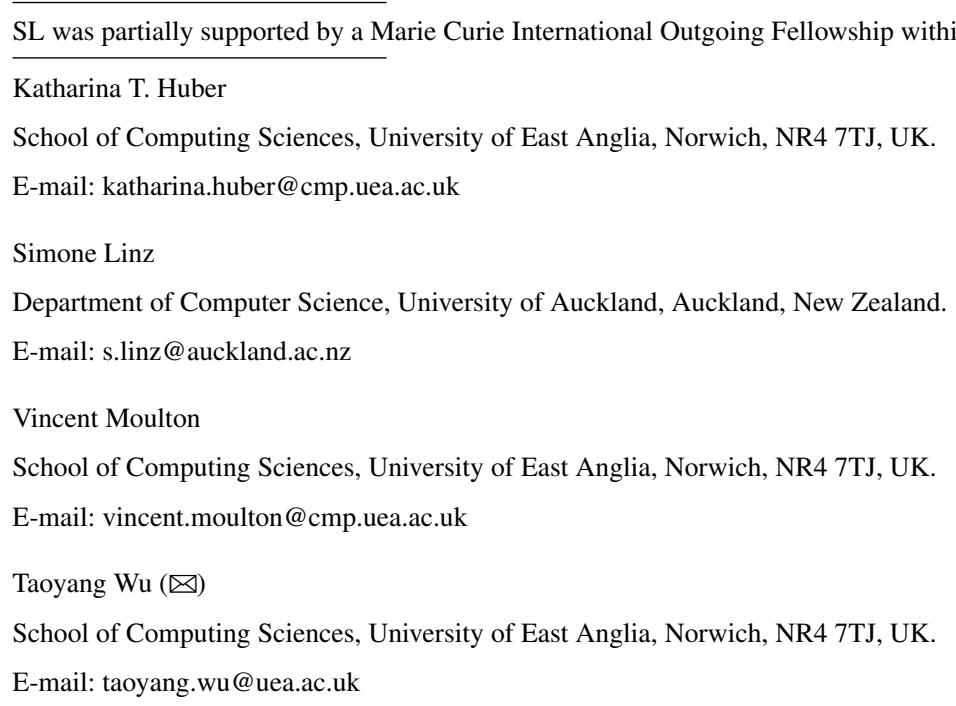




\section{Introduction}

Phylogenetic networks are a generalization of evolutionary or phylogenetic trees that are used to represent the evolution of species which have undergone reticulate evolution (Huson et al, 2010). Mathematically speaking, such a network is a binary, directed, acyclic graph that has a single root, no indegree- and outdegree-1 vertices, and whose leaf set corresponds to the set of species of interest. We shall also consider unrooted networks, which are defined in a similar manner, except that the underlying graphs are undirected (see Section 2 for full definitions). Phylogenetic networks allow the representation of speciation events as well as events in which two species come together to form a new species (through biological processes such as hybridization or recombination).

Several types of phylogenetic networks have been defined and various methods have been proposed for their construction (see e.g. Huson et al, 2010; Gusfield, 2014). In Strimmer and Moulton (2000) it was proposed to develop methods to build networks by searching through networks so as to optimize likelihood, and some progress has been made in this direction for this and related criteria (see e.g. Jin et al, 2006, 2007; Radice, 2011), especially in the recent work by Yu et al (2014). However, although a similar strategy has been successfully employed for several years to search tree space and construct phylogenetic trees (see e.g. Lemey et al, 2009; Swofford et al, 1996), the mathematical underpinnings necessary to understand how to systematically search through spaces of phylogenetic networks have yet to be fully developed.

In this paper, we will take some first steps in the development of this theory. In particular, we introduce certain operations on phylogenetic networks called local subnetwork transfer (LST) operations and investigate their properties for some spaces of relatively simple phylogenetic networks. The LST operations that we define generalize and extend the nearest-neighbor interchange (NNI) operations on phylogenetic trees (Robinson, 1971; Moore et al, 1973), whose properties are well-studied and which are commonly used in computer programs that search for optimal trees in tree space (Swofford et al, 1996). We will mainly concentrate on properties of LST operations when they are applied to unrooted and rooted level-1 networks (Jansson et al, 2006; Gambette et al, 2009), a well understood class of phylogenetic networks that are almost tree-like, and which have been used to, for example, represent the evolution of viruses (Huber et al, 2011).

We now describe the contents of the rest of this paper. After recalling some preliminaries on phylogenetic networks in the next section, in Section 3 we define LST operations on general unrooted phylogenetic networks. We then show in Section 4 that such operations can be used to convert any unrooted level-1 network into any other such that each intermediate network is also level-1 (see Theorem 2). In Fig. 1 we illustrate this result for the case where all of the networks in question are on the same set of four species (note that similar graphs for phylogenetic trees appear in e.g. Billera et al, 2001). In this space of networks, the vertices represent each possible unrooted level-1 network, and each edge represents the fact that the two incident networks can be converted into one another using precisely one LST operation. In particular, the fact that this graph is connected is equivalent to the fact that we can convert any network on four leaves into any other such network by using a sequence of LST operations.

Various related questions naturally arise when considering network spaces such as that pictured in Fig. 1. One concerns the size of the neighborhood of any network, that is, the number of networks adjacent to this one. For example, each network in the bottom of Fig. 1 has a neighborhood of size six. In Section 5, we give an explicit formula for the size of such a neighborhood for unrooted level-1 networks in general (see Theorem 3). In contrast to the NNI neighborhood 


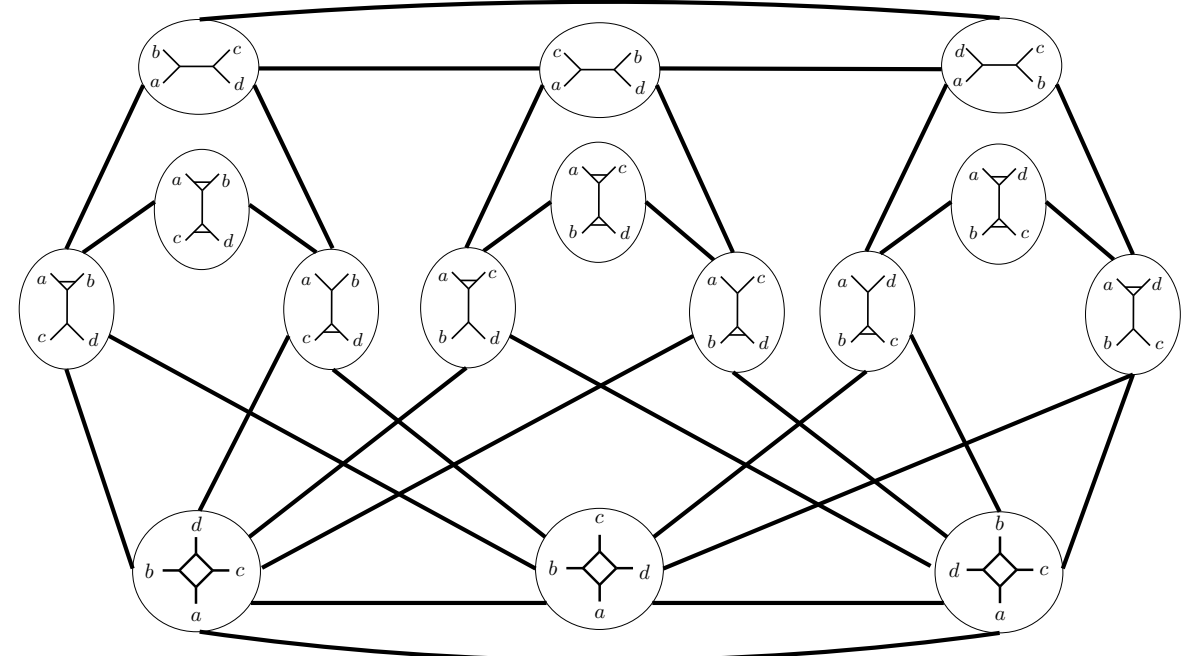

Fig. 1 The space of unrooted level-1 networks each having leaf set $\{a, b, c, d\}$. Each network is represented by a node, and two networks are connected by an edge in bold if and only if one can be converted into the other by one LST operation.

of a phylogenetic tree which only depends on the number of leaves in the given tree (Robinson, 1971), our formula also depends on the structure of the network in question, as it requires knowledge of parameters such as the number of 3- and 4-cycles. We therefore also investigate in what way the size of the neighborhood of a network depends on the network, and, more specifically, we give a description of those networks that have the smallest neighborhoods for unrooted level-1 networks (see Theorem 4).

It is also natural to try to understand properties of the metric on unrooted level-1 networks that is induced by LST operations, which we shall call the LST metric for short. This metric corresponds to determining the minimum number of LST operations that are required to convert one level-1 network into another one. For example, in Fig. 1, the LST metric between the networks in the top left and bottom right of the diagram is three, which corresponds to the length of a shortest path connecting these two nodes. In Section 6 we give some upper and lower bounds on the diameter of the LST metric, that is, bounds on the largest value that this metric can take (e.g. the diameter of the LST metric given by the graph depicted in Fig. 1 is four). Note that for phylogenetic trees, this metric is analogous to the NNI metric (Day, 1983), for which asymptotical upper and lower diameter bounds have been determined (Li et al, 1996).

In Section 7, we adapt the LST operations so that they can be applied to rooted level-1 networks. This is somewhat more complicated as more cases need to be taken into account to handle the directions of the arcs and also for dealing with the root. In particular, we prove that we can convert any rooted level-1 network into any other through a sequence of LST operations (see Theorem 6). In the final section, we conclude with a discussion of some possible future directions.

\section{Preliminaries}

We shall mainly follow the notation presented in Gambette et al (2012). We let $X$ denote a set of taxa of cardinality $n \geq 2$ and assume that $k$ is a non-negative integer.

Given an undirected graph $G$ we let $V(G)$ and $E(G)$ denote the vertex set and the edge set of $G$, respectively. Note that we consider an edge of $G$ as a 2-element set of $V(G)$. A biconnected component of a graph is a maximal induced subgraph without any cut vertex (that is, a vertex whose removal disconnects the graph). A cut edge in a graph is an 
edge whose removal disconnects the graph. Each edge that is not incident with a leaf is referred to as an interior edge. Similarly, each vertex that is not a leaf is referred to as an interior vertex. A vertex is called a tree vertex if it is an interior vertex that is not contained in a cycle. The blobs of an undirected graph are its maximal connected components without cut edges. Note that the blobs are not the biconnected components of the graph as there is no blob with precisely two vertices. A blob is trivial if it contains precisely one vertex, and non-trivial otherwise. A chord of a graph $G$ is an edge $e$ in $G$ such that there is some cycle in $G$ which does not contain $e$ but which contains both vertices of $e$. A link in $G$ is an edge $\{u, v\}$ in $G$ such that there exist two vertex disjoint cycles of $G$ that contain $u$ and $v$, respectively. Finally, a graph $G$ is called level-k if a spanning tree of $G$, that is, an unrooted tree connecting all vertices of $G$, can be obtained by removing at most $k$ edges per blob.

Given an undirected graph $G$, we let $b(G)$ denote the number of non-trivial blobs in $G, b_{3}(G)$ the number of blobs in $G$ consisting of precisely one 3-cycle, $b_{4}(G)$ the number of blobs consisting of precisely one 4-cycle, and $t(G)$ the number of tree vertices in $G$.

An unrooted phylogenetic network $N$ on $X$ is a loopless connected graph without multi-edges whose vertices have either degree 3 (interior vertices), or degree 1 (the leaves), and such that its set of leaves is equal to $X$. In the following, we often refer to an unrooted phylogenetic network on $X$ as a network on $X$, or simply as a network if the leaf set is not of importance. A network $N$ is called level- $k$ if it is level- $k$ as a graph. Thus, a phylogenetic tree is simply a level-0 network; and a level-1 network is sometimes also referred to as a galled-tree (see, e.g. Semple and Steel, 2006; Rosselló and Valiente, 2009). A subcomponent of $N$ is a connected component of the graph obtained by removing a cut edge $e$ of $N$. In this case, we also say that the subcomponent is incident with the vertex in $e$ that is not contained in the subcomponent. Two subcomponents are disjoint if they share no common vertices. Two networks $N$ and $N^{\prime}$ on $X$ are isomorphic if there exists a graph isomorphism between $N$ and $N^{\prime}$ that is the identity on the leaves.

To work with unrooted networks, it will also be helpful to consider quartets (see, e.g. Gambette et al, 2012; Keijsper and Pendavingh, 2014) and some related concepts which we define as follows. Suppose $|X| \geq 4$. An unordered pair of 2element subsets $\{\{a, b\},\{c, d\}\}$ is called a quartet of $X$, denoted by $a b \mid c d$, if $a, b, c, d$ are four pairwise distinct elements of $X$. A quartet $a b \mid c d$ is consistent with a network $N$ on $X$ if $\{a, b, c, d\} \subseteq X$ and there exist vertex disjoint paths in $N$ that connect $a$ to $b$ and $c$ to $d$, respectively. The set of quartets consistent with $N$ is denoted by $\mathscr{Q}(N)$. Note that if there exist two disjoint subcomponents of $N$, one containing $a$ and $b$ and the other containing $c$ and $d$, then $a b \mid c d \in \mathscr{Q}(N)$. In addition, if $N$ is isomorphic to another network $N^{\prime}$ on $X$, then $\mathscr{Q}(N)=\mathscr{Q}\left(N^{\prime}\right)$. Note that the converse of this observation is generally not true (see, e.g., Lemma 2 in Keijsper and Pendavingh, 2014, for a related result on level-1 networks).

We next present two elementary results concerning level-1 networks.

Lemma 1 If $N$ is a level-1 network with $n \geq 2$ leaves, then the number of vertices of $N$ is $2 n-2+2 b(N)$ and the number of edges of $N$ is $2 n-3+3 b(N)$.

Proof Let $N$ be as in the statement of the lemma and put $k=b(N)$. The lemma clearly holds for $k=0$ since any phylogenetic tree on $n$ leaves has $2 n-2$ vertices and $2 n-3$ edges (see e.g. (Semple and Steel, 2003, p.17)). Thus, we may assume for the remainder of the proof that $k \geq 1$. Let $C_{1}, \ldots, C_{k}$ be the set of cycles in $N$ (or, equivalently, the set of non-trivial blobs of $N$ as $N$ is level-1). Fix an edge $e_{i}=\left\{u_{i}, v_{i}\right\}$ in cycle $C_{i}$ for $1 \leq i \leq k$ and let $V_{0}=\left\{u_{1}, v_{1}, \ldots, u_{k}, v_{k}\right\}$. Since $N$ is level- $1, e_{i} \cap e_{j}=\emptyset$ for all $1 \leq i<j \leq k$. Moreover, deleting the edges $\left\{e_{1}, \ldots, e_{k}\right\}$ from $N$ results in a tree $T$ with the same $n$ leaves as $N$. Consider the phylogenetic tree $T^{\prime}$ obtained from $T$ by suppressing all vertices in $V_{0}$. Then 


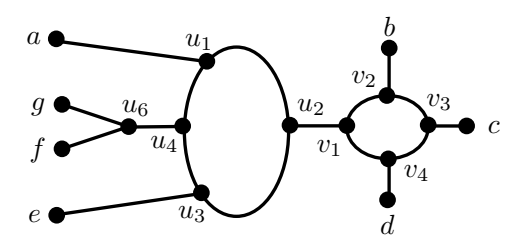

(i)

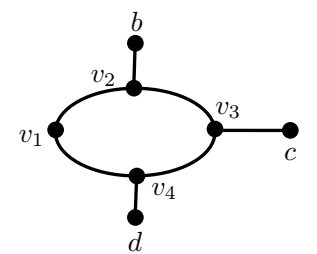

(ii)

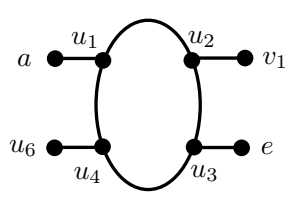

(iii)

Fig. 2 Example of a subcomponent decomposition. The network $N$ in (i) has a subcomponent decomposition $\left[U_{0}, \ldots, U_{4}\right]$ with $U_{0}=\left\{u_{1}, u_{2}, u_{3}, u_{4}\right\}$, $U_{1}=\left\{v_{1}, v_{2}, v_{3}, v_{4}, b, c, d\right\}, U_{2}=\{e\}, U_{3}=\left\{u_{6}, g, f\right\}$, and $U_{4}=\{a\}$. The subcomponent $N\left[U_{1}\right]$ and the subgraph $\widetilde{N}\left[U_{0}\right]$ are depicted in (ii) and (iii), respectively.

$T^{\prime}$ contains $2 n-2$ vertices and $2 n-3$ edges. Thus we have

$$
|V(N)|=\left|V\left(T^{\prime}\right)\right|+2 k=2 n-2+2 k \text { and }|E(N)|=\left|E\left(T^{\prime}\right)\right|-2 k+5 k=2 n-3-3 k,
$$

as required.

Lemma 2 Suppose that $N$ is a level-1 network. Then the following two statements hold:

(i) $N$ does not contain a chord.

(ii) Each link in $N$ is a cut edge. In particular, it is an interior edge that is not contained in any cycle of $N$.

Proof (i) Assume for contradiction that $N$ contains a chord $e=\{u, v\}, u, v \in V(N)$, and let $C$ be a cycle of $N$ that contains both $u$ and $v$, but not $e$. Denote the blob of $N$ that contains $C$ by $H$. Then $H$ is a non-trivial blob which contains $e$. This implies that obtaining a spanning tree of $N$ requires deleting at least two edges of $H$, a contradiction to $N$ being level-1.

(ii) Suppose $v_{1}, v_{2} \in V(N)$ such that $e=\left\{v_{1}, v_{2}\right\}$ is a link. It suffices to show that $e$ is a cut edge of $N$ because this implies that $e$ is an interior edge not contained in any cycle of $N$. To this end, let $C_{1}$ and $C_{2}$ be two vertex disjoint cycles in $N$ such that $v_{i}$ is contained in $C_{i}$ for $i=1,2$. If $e$ were not a cut edge, then $C_{1}$ and $C_{2}$ would be contained in the same non-trivial blob $H_{e}$ of $N$. Since obtaining a spanning tree of $N$ requires deleting at least two edges of $H_{e}$ (one from $C_{1}$ and the other from $C_{2}$ ), this is impossible as $N$ is level-1.

Analogous to tree decompositions used in the study of tree operations and tree metrics (see, e.g., Allen and Steel, 2001; Moulton and $\mathrm{Wu}, 2015)$, we next introduce a decomposition of a network that will be used later. Given a network $N$ and a subset $U \subseteq V(N)$, let $N[U]$ be the subgraph of $N$ on $U$ whose edges are precisely the edges $\{u, v\} \in E(N)$ with $\{u, v\} \subseteq U$. Furthermore, let $\widetilde{N}[U]$ denote the subgraph obtained from $N[U]$ by adding, for all edges $\{u, v\} \in E(N)$ with $u \in U$ and $v \notin U$, the vertex $v$ and the edge $\{u, v\}$. A list $\left[U_{0}, U_{1}, \ldots, U_{m}\right](m \geq 1)$ of non-empty subsets of $V(N)$ is a subcomponent decomposition of $N$ if the following three conditions hold: (i) $U_{i} \cap U_{j}=\emptyset$ for $0 \leq i<j \leq m$ and $V(N)=U_{0} \cup U_{1} \cup \cdots \cup U_{m}$, (ii) $N\left[U_{i}\right]$ is a subcomponent of $N$ for $1 \leq i \leq m$, and (iii) $N\left[U_{0}\right]$ is a connected subgraph of $N$ (see Fig. 2 for an example).

We end this section with the following result concerning subcomponent decompositions of networks.

Theorem 1 Suppose that $N$ is a network and $\left[U_{0}, U_{1}, \ldots, U_{m}\right]$ is a subcomponent decomposition of $N$. Then the following three statements hold.

(i) $C$ is a cycle in $N$ if and only if there exists some (necessarily unique) $0 \leq i \leq m$ such that $C$ is a cycle in $N\left[U_{i}\right]$. 
(ii) A subgraph $K$ of $N$ is a non-trivial blob in $N$ if and only if there exists some (necessarily unique) $0 \leq i \leq m$ such that $K$ is a non-trivial blob in $N\left[U_{i}\right]$.

(iii) $N$ is level-k if and only if $N\left[U_{i}\right]$ is level- $k$, for all $0 \leq i \leq m$.

In addition, we have

$$
b(N)=\sum_{0 \leq i \leq m} b\left(N\left[U_{i}\right]\right) \quad \text { and } \quad t(N)=t\left(\tilde{N}\left[U_{0}\right]\right)+\sum_{1 \leq i \leq m} t\left(N\left[U_{i}\right]\right)
$$

Proof (i) This is a consequence of (ii) since each cycle of a graph is contained in a (necessarily unique) non-trivial blob of that graph.

To see the remaining statements of the theorem note first that since $\left[U_{0}, U_{1}, \ldots, U_{m}\right]$ is a subcomponent decomposition of $N$, there exists, for all $1 \leq i \leq m$, a cut edge $e_{i}$ of $N$ whose removal induces $N\left[U_{i}\right]$. Put $E^{\prime}=\left\{e_{1}, \ldots, e_{m}\right\}$. Note next that, for all $1 \leq i<j \leq m$, we have $e_{i} \neq e_{j}$ because if there existed distinct $i$ and $j$ such that $e_{i}=e_{j}$ then $V(N)=U_{i} \cup U_{j}$ would follow in contradiction to $U_{0} \neq \emptyset$.

(ii) Clearly, removing all edges in $E^{\prime}$ from $N$ results in a graph with $m+1$ connected components and these are $N\left[U_{i}\right]$, for $0 \leq i \leq m$. Since non-trivial blobs do not contain a cut edge, it follows that a subgraph $K$ of $N$ is a non-trivial blob in $N$ if and only if $K$ is a non-trivial blob in $N\left[U_{i}\right]$ for a (necessarily unique) index $i$ in $\{0, \ldots, m\}$.

(iii) Suppose first that $N$ is level- $k$. Then there exists a set of edges $E^{*}$ of $N$ such that removing all edges in $E^{*}$ from $N$ yields a spanning tree of $N$ and each non-trivial blob contains at most $k$ edges in $E^{*}$. Note that $E^{*}$ and $E^{\prime}$ are necessarily disjoint as each edge in $E^{\prime}$ is a cut edge. Hence, removing for $0 \leq i \leq m$, the edges in $E^{*} \cap E\left(N\left[U_{i}\right]\right)$ results in a spanning tree of $N\left[U_{i}\right]$ and each non-trivial blob in $N\left[U_{i}\right]$ contains at most $k$ edges in $E^{*} \cap E\left(N\left[U_{i}\right]\right)$. Thus, $N\left[U_{i}\right]$ is level- $k$ for all $0 \leq i \leq m$.

Conversely, suppose that, for all $0 \leq i \leq m$, the subgraphs $N\left[U_{i}\right]$ are level- $k$. For all $0 \leq i \leq m$, let $E_{i}^{*}$ denote a set of edges of $N\left[U_{i}\right]$ such that removing all edges in $E_{i}^{*}$ from $N\left[U_{i}\right]$ results in a spanning tree of $N\left[U_{i}\right]$ and each non-trivial blob in $N\left[U_{i}\right]$ contains at most $k$ edges in $E_{i}^{*}$. Now let $E^{*}=\bigcup_{0 \leq i \leq m} E_{i}^{*}$. Then a spanning tree of $N$ can be obtained by removing all edges in $E^{*}$ from $N$. Since each non-trivial blob $H$ of $N$ is contained in $N\left[U_{i}\right]$ for some $0 \leq i \leq m$ it follows that $H$ contains at most $k$ edges in $E^{*}$. Hence, $N$ is also level- $k$.

It remains to prove the two equations in Eq. (1). Since the equality concerning $b(N)$ follows from (ii), it suffices to prove the second one. To this end, consider an arbitrary vertex $v$ in $N$. Let $0 \leq j \leq m$ such that $v \in U_{j}$. Then $v$ is an interior vertex in $N$ if and only if $v$ is either an interior vertex of $\widetilde{N}\left[U_{0}\right]$ (i. e., $j=0$ ) or an interior vertex of $N\left[U_{j}\right]$ (i. e., $j \geq 1$ ). This implies $v$ is a tree vertex in $N$ if and only if $v$ is either a tree vertex in $\widetilde{N}\left[U_{0}\right]$ or a tree vertex in $N\left[U_{j}\right]$ (but not both). The equality in Eq. (1) concerning $t(N)$ now follows.

\section{Local subnetwork transfer operations on unrooted networks}

In this section we introduce our local operations on phylogenetic networks. For the following, let $N$ be a phylogenetic network on $X$. We begin by defining a generalization of the NNI operation for trees.

Nearest-neighbor interchange (NNI) operation: Let $\theta=\left(e_{1}, e, e_{2}\right)$ be an ordered triple of edges in $N$ such that $e_{1}, e, e_{2}$ is a path in $N$ (that is, $e_{1} \cap e \neq \emptyset \neq e_{2} \cap e$ while $e_{1} \cap e_{2} \cap e=\emptyset$ ), $e$ is neither a link nor a chord in $N$, and $e$ is not contained in a 3-cycle. Denoting by $\Sigma(N)$ the set of all such triples, we associate to each $\theta=\left(e_{1}, e, e_{2}\right) \in \Sigma(N)$ the phylogenetic 


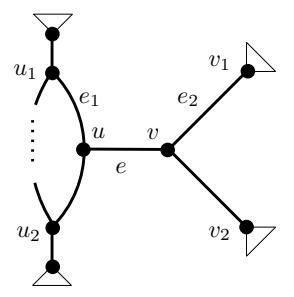

(i)

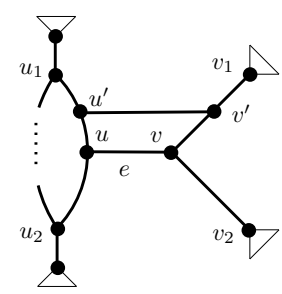

(ii)

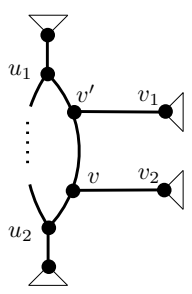

(iii)

Fig. 3 Two networks that are one NNI operation apart: the network in (iii) can be obtained from the one in (i) with one NNI operation on $e$, as indicated by (ii).

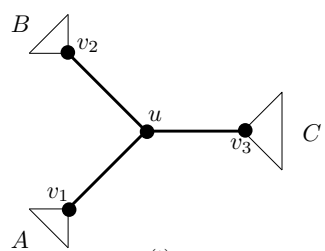

(i)

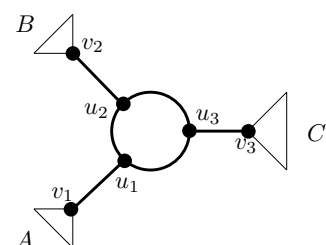

(ii)

Fig. 4 Two networks that are one TC operation apart: The network in (ii) can be obtained from the one in (i) with one TC-pop on $u$, while the network in (i) can be obtained from the one in (ii) with one TC-shrink on the cycle $\left\{u_{1}, u_{2}, u_{3}\right\}$.

network $\theta(N)$ defined as follows. Let $e=\{u, v\}$ with $u \in e_{1}$ and $v \in e_{2}$ and subdivide $e_{1}$ and $e_{2}$ with two new vertices $u^{\prime}$ and $v^{\prime}$, respectively. Then $\theta(N)$ is obtained from $N$ by connecting $u^{\prime}$ and $v^{\prime}$ by an edge, deleting the edge $\left\{u^{\prime}, u\right\}$, and suppressing the resulting degree two vertices (i.e., $u^{\prime}$ and $u$ ) (see Fig. 3). We say that $\theta(N)$ is obtained from $N$ by applying the NNI operation $\theta$ on $e$ and refer to the edge $e$ as the axis of $\theta$.

Note that if $N$ is a phylogenetic tree, then this operation is equivalent to the classical NNI operation (see (Moore et al, 1973; Robinson, 1971) and also e.g. (Humphries and Wu, 2013) for more recent results). In addition, the order of the three edges in $\theta$ is important as changing it can alter the network that is obtained by applying $\theta$. Note that the condition that the axis of a triple is not contained in a 3-cycle of $N$ ensures that $\theta(N)$ does not contain a multi-edge, and hence that $\theta(N)$ is a phylogenetic network. In addition, we assume that $e$ is not a link in the definition of an NNI operation as otherwise the level of $\theta(N)$ could be different from that of $N$. Finally, the reason for not allowing $e$ to be a chord is to make sure that NNI operations are reversible, as we shall explain below.

We now describe two operations which allow us to convert networks between different levels.

Three-cycle (TC) operations: Let $u$ be an interior vertex of $N$ that is not contained in a cycle of $N$. Furthermore, let $v_{1}, v_{2}$ and $v_{3}$ be the vertices in $N$ that are adjacent to $u$. We obtain a network $N^{\prime}$ on $X$ from $N$ by removing vertex $u$ and edges $\left\{u, v_{1}\right\},\left\{u, v_{2}\right\}$ and $\left\{u, v_{3}\right\}$ and inserting new vertices $u_{1}, u_{2}$ and $u_{3}$ and new edges $\left\{u_{1}, v_{1}\right\},\left\{u_{2}, v_{2}\right\},\left\{u_{3}, v_{3}\right\}$, $\left\{u_{1}, u_{2}\right\},\left\{u_{2}, u_{3}\right\}$, and $\left\{u_{3}, u_{1}\right\}$ (see Fig. 4). We say that $N^{\prime}$ is obtained from $N$ by a three-cycle pop on $u$ (or TC-pop, for short). Note that $N^{\prime}$ is a phylogenetic network with one more cycle than $N$. Conversely, suppose that $C=\left\{u_{1}, u_{2}, u_{3}\right\}$ is a cycle in $N$ of length 3 and that $\left\{u_{1}, v_{1}\right\},\left\{u_{2}, v_{2}\right\}$, and $\left\{u_{3}, v_{3}\right\}$ are edges in $N$ for which $\left|\left\{u_{1}, u_{2}, u_{3}, v_{1}, v_{2}, v_{3}\right\}\right|=6$ holds. Then we obtain a phylogenetic network $N^{\prime}$ on $X$ from $N$ by removing vertices $u_{1}, u_{2}, u_{3}$ and the six edges incident with any one of these three vertices, inserting a new vertex $u$ and new edges $\left\{u, v_{1}\right\},\left\{u, v_{2}\right\}$ and $\left\{u, v_{3}\right\}$, and then suppressing any vertices of degree 2 that might result. We say that $N^{\prime}$ is obtained from $N$ by a three-cycle shrink of $C$ (or TC-shrink, for short). Note that $N^{\prime}$ is a phylogenetic network with one less cycle than $N$ (see Fig. 4). If a network $N^{\prime}$ on $X$ can be 
obtained from $N$ by either a TC-pop, a TC-shrink, or an NNI operation, then we say that $N^{\prime}$ can be obtained from $N$ by a local subnetwork transfer (LST) operation.

Note that if we apply first a TC-pop on $N$ and then a TC-shrink of the resulting 3-cycle, or a TC-shrink and then a TC-pop on the resulting vertex, then we obtain a network that is isomorphic to $N$. Thus, these two operations are reversible. This property is desirable, as it is convenient for defining metrics on trees or networks in terms of operations (see also Section 6). We now show that, just as with phylogenetic trees, NNI operations can also be reversed.

Lemma 3 Suppose that $N$ is a phylogenetic network on $X$ and that $\theta$ is an NNI operation on $N$. Then there exists an NNI operation $\theta^{\prime}$ on $\theta(N)$ such that $N=\theta^{\prime}(\theta(N))$.

Proof Let $e=\{u, v\}, u, v \in V(N)$, denote the axis of $\theta$ and set $N^{\prime}=\theta(N)$. Since $\theta$ is an NNI operation on $e$, there exist four distinct vertices $u_{1}, v_{1}, u_{2}, v_{2}$ in $V(N)-\{u, v\}$ and four edges $e_{1}=\left\{u_{1}, u\right\}, e_{2}=\left\{v_{1}, v\right\}, e_{3}=\left\{u_{2}, u\right\}$, and $e_{4}=\left\{v_{2}, v\right\}$ in $N$ such that $\theta=\left(e_{1}, e, e_{2}\right)$. Let $u^{\prime}$ and $v^{\prime}$ be the vertices that divide $e_{1}$ and $e_{2}$ in the construction of $N^{\prime}$ (see Fig. 3 for an illustration). Then $e_{1}^{\prime}=\left\{u_{1}, v^{\prime}\right\}, e^{\prime}=\left\{v^{\prime}, v\right\}, e_{2}^{\prime}=\left\{v_{1}, v^{\prime}\right\}$ and $e_{3}^{\prime}=\left\{v, u_{2}\right\}$ are edges in $N^{\prime}$, and we have

$$
V\left(N^{\prime}\right)=(V(N)-\{u\}) \cup\left\{v^{\prime}\right\} \quad \text { and } \quad E\left(N^{\prime}\right)=\left(E(N)-\left\{e_{1}, e_{2}, e_{3}, e\right\}\right) \cup\left\{e_{1}^{\prime}, e_{2}^{\prime}, e_{3}^{\prime}, e^{\prime}\right\}
$$

In order to construct the required operation $\theta^{\prime}$, we consider the edge $e^{\prime}$ which is clearly an interior edge of $N^{\prime}$. Since $u_{1}, u_{2}, v_{1}, v_{2}$ are four distinct vertices, $e^{\prime}$ is clearly not contained in any 3 -cycle of $N^{\prime}$. We claim first that $e^{\prime}$ is not a link in $N^{\prime}$. Indeed, if $e^{\prime}$ were a link in $N^{\prime}$, then there would exist two vertex disjoint cycles $C_{1}$ and $C_{2}$ in $N^{\prime}$ with $v^{\prime} \in V\left(C_{1}\right)$ and $v \in V\left(C_{2}\right)$. Let $P_{1}$ be the path between $u_{1}$ and $v_{1}$ obtained from $C_{1}$ by removing $v^{\prime}$ and the two edges incident with $v^{\prime}$. Then $P_{1}$ is also a path in $N$ because it contains neither $v$ nor $v^{\prime}$. Similarly, the path $P_{2}$ between $u_{2}$ and $v_{2}$ obtained from $C_{2}$ by removing $v$ and its incident edges is also a path in $N$. As $P_{1}$ and $P_{2}$ are vertex disjoint, the cycle $C$ in $N$ consisting of $P_{1}, P_{2}$ and the four edges $e_{1}, e_{2}, e_{3}, e_{4}$ contains vertex $u$ and $v$, but not $e$. This is a contradiction to the assumption that $e$ is not a chord in $N$, and hence the claim follows.

We claim next that $e^{\prime}$ is not a chord in $N^{\prime}$. If $e^{\prime}$ were a chord in $N^{\prime}$ then, by construction and the fact that each vertex in $N^{\prime}$ has degree three or one, there would exist a cycle $C$ in $N^{\prime}$ with $\left\{e_{1}^{\prime}, e_{2}^{\prime}, e_{3}^{\prime}, e_{4}\right\} \subseteq E(C)$ and $e^{\prime} \notin E(C)$. Let $P_{1}$ be the path in $N^{\prime}$ between $v_{1}$ and $v_{2}$ induced by $C$ such that $e_{1}^{\prime} \in E\left(P_{1}\right)$ (and hence also $e_{2}^{\prime} \in E\left(P_{1}\right)$ ). Let $P_{2}$ be the path in $N^{\prime}$ obtained from $C$ by removing all edges contained in $P_{1}$. Thus, $P_{2}$ joins $v_{1}$ and $v_{2}$ and $V\left(P_{1}\right) \cap V\left(P_{2}\right)=\left\{v_{1}, v_{2}\right\}$. Since when starting at $v^{\prime}$ and traversing $P_{1}$ we either encounter $u_{2}$ first or $u_{1}$ we need to consider the following two cases.

(i) $e_{3}^{\prime} \in E\left(P_{1}\right)$ (and hence also $e_{4} \in E\left(P_{1}\right)$ ). Let $P_{1}^{*}$ be the path between $u_{1}$ to $u_{2}$ obtained from $P_{1}$ by deleting the edges $e_{1}^{\prime}, e_{2}^{\prime}, e_{3}^{\prime}, e_{4}$. Then $V\left(P_{1}^{*}\right) \cap V\left(P_{2}\right)=\emptyset$. Noting that $\left(V\left(P_{1}^{*}\right) \cup V\left(P_{2}\right)\right) \cap\left\{v, v^{\prime}\right\}=\emptyset$, it follows that the two paths $P_{1}^{*}$ and $P_{2}$ can be regarded as two vertex disjoint paths in $N$. Let $C_{1}$ be the cycle in $N$ obtained from $P_{1}^{*}$ by adding the edges $e_{1}$ and $e_{3}$, and let $C_{2}$ be the cycle in $N$ obtained from $P_{2}$ by adding the edges $e_{2}$ and $e_{4}$. Then $C_{1}$ and $C_{2}$ are two vertex disjoint cycles in $N$ and $u$ is a vertex in $C_{1}$ and $v$ is a vertex in $C_{2}$. Thus, $e$ is a link in $N$ which is impossible.

(ii) $e_{3}^{\prime} \notin E\left(P_{1}\right)$ (and hence also $e_{4} \notin E\left(P_{1}\right)$ ). Then $V\left(P_{2}\right)=V(C)-V\left(P_{1}\right)$ implies that $e_{3}^{\prime}$ and $e_{4}$ are edges of $P_{2}$. Therefore, the subgraph $P_{1}^{\prime}$ of $N^{\prime}$ obtained from $P_{1}$ by deleting $e_{1}^{\prime}$ and $e_{2}^{\prime}$ is a path from $u_{1}$ to $v_{2}$, and the subgraph $P_{2}^{\prime}$ of $N^{\prime}$ obtained from $P_{2}$ by deleting $e_{3}^{\prime}$ and $e_{4}$ is a path from $u_{2}$ to $v_{1}$. Moreover, $P_{1}^{\prime}$ and $P_{2}^{\prime}$ are vertex disjoint and neither of them contains a vertex in $\left\{v, v^{\prime}\right\}$. Hence $P_{1}^{\prime}$ and $P_{2}^{\prime}$ are also two vertex disjoint paths in $N$. Let $C$ be the subgraph of $N$ consisting of 
the two paths $P_{1}^{\prime}, P_{2}^{\prime}$ and the edges $e_{i}$ for $1 \leq i \leq 4$. Then $C$ is a cycle in $N$ such that $\{u, v\} \subseteq V(C)$. Hence, $e \in E(C)$, a contradiction to the assumption that $e$ is not a chord in $N$. Thus, $e^{\prime}$ cannot be a chord of $N^{\prime}$ either, as claimed.

In summary, we have that $e^{\prime}$ is an interior edge of $N^{\prime}$ that is neither contained in a 3-cycle of $N$ nor is a link or a chord of $N^{\prime}$. Thus, $\theta^{\prime}=\left(e_{1}^{\prime}, e^{\prime}, e_{3}^{\prime}\right)$ is an NNI operation on $N^{\prime}$. The lemma follows as $N$ is isomorphic to $\theta^{\prime}\left(N^{\prime}\right)$.

\section{LST operations on unrooted level-1 networks}

In this section, we shown that it is possible to convert any level-1 network into any other such network through a sequence of LST operations. Let $N$ denote a level-1 network. For clarity of exposition, we divide the NNI operations on $N$ into the following three types according to their axes. More specifically, an NNI operation $\theta$ with axis $e=\{u, v\}$ in $N$ is type I if both $u$ and $v$ are tree vertices, type II if precisely one of $u$ or $v$ is a tree vertex, and type III otherwise (see Fig. 5, Fig. 6, and Fig. 7 for an illustration). Since $e$ is not a link, it follows that $\theta$ is type III if and only if neither $u$ nor $v$ is a tree vertex which, by Lemma 2, is equivalent to $e$ being contained in a cycle of $N$. This observation allows us to further divide type III operations into two subtypes as follows. A type III NNI operation $\left(e_{1}, e, e_{2}\right)$ is type III-A if precisely one of the edges in $\left\{e_{1}, e_{2}\right\}$ is contained in the cycle containing $e$, and type III-B otherwise.
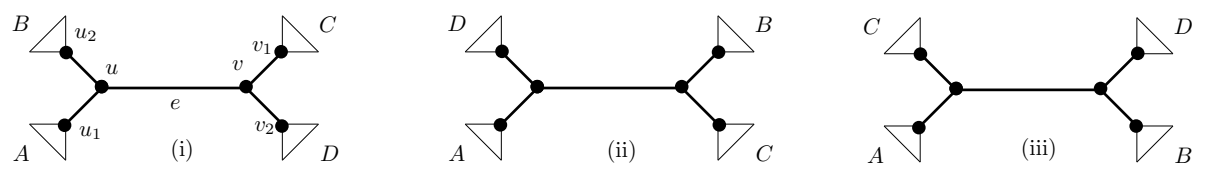

Fig. 5 A schematic representation of a type I NNI operation. Here the networks in (ii) and (iii) can be obtained from the network in (i) by one type I NNI operation on $e$.



(i)

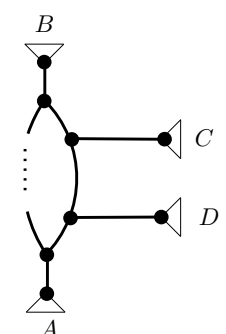

(ii)

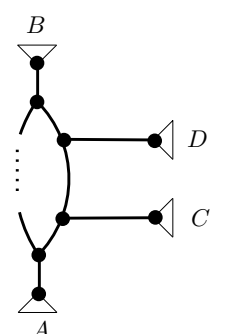

(iii)

Fig. 6 A schematic representation of a type II NNI operation. Here the networks in (ii) and (iii) can be obtained from the network in (i) by one type II NNI operation on $e$.

Recall that for a level-1 network $N$, the number of non-trivial blobs of $N$ is denoted by $b(N)$ and the number of tree vertices in $N$ is denoted by $t(N)$. The following lemma characterizes the changes of these two quantities when an NNI operation is applied. 


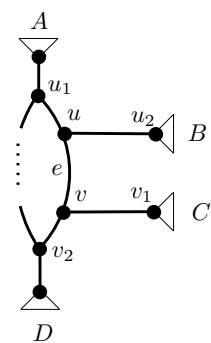

(i)

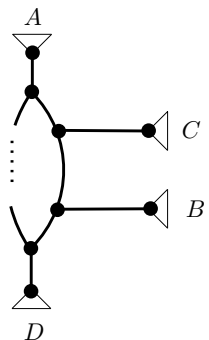

(ii)

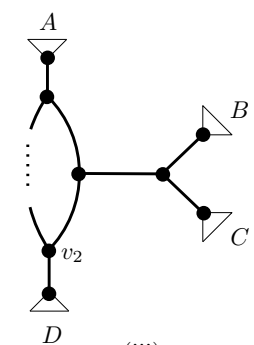

(iii)

Fig. 7 A schematic representation of a type III NNI operation. Here the networks in (ii) and (iii) can be obtained from the network in (i) by a type III-A and type III-B NNI operation on $e$, respectively.

Lemma 4 Suppose that $N$ is a level-1 network and $\theta$ is an NNI operation on $N$. Then $\theta(N)$ is a level-1 network with $b(\theta(N))=b(N)$ holding. In addition, we have

$$
t(\theta(N))= \begin{cases}t(N) & \text { if } \theta \text { is type I or type III-A, } \\ t(N)-1 & \text { if } \theta \text { is type } \mathrm{II} \\ t(N)+1 & \text { if } \theta \text { is type } \mathrm{III}-\mathrm{B} .\end{cases}
$$

Proof Let $e=\{u, v\}, u, v \in V(N)$, denote the axis of $\theta$. Since there are three types of NNI-operations, we have three cases to consider. In each case we specify four disjoint subcomponents in $N$ which are also disjoint subcomponents in $\theta(N)$. For clarity of exposition, through the proof we denote those components by $A, B, C, D$, and use the notation that $V_{1}=V(A), V_{2}=V(B), V_{3}=V(C), V_{4}=V(D), V_{0}=V(N) \backslash \cup_{1 \leq i \leq 4} V_{i}$ and $V_{0}^{\prime}=V(\theta(N)) \backslash \cup_{1 \leq i \leq 4} V_{i}$. Moreover, we denote the two vertices adjacent with $u$ and $v$, respectively, by $u_{1}, u_{2}, v_{1}$ and $v_{2}$, as indicated in Fig 5(i), Fig 6(i) and Fig. 7(i).

Case I: $\theta$ is of type I. Then both $u$ and $v$ are tree vertices, and removing $e$ and the other edges incident with $u$ and $v$ results in four subcomponents $A, B, C, D$ of $N$ (see Fig. 5(i)). Since $\theta$ swaps these four subcomponents, it follows that $\theta(N)$ is isomorphic to either the network depicted in Fig. 5(ii) or the one in Fig. 5(iii). Since $N\left[V_{0}\right]$ and $\theta(N)\left[V_{0}^{\prime}\right]$ both comprise of only one edge, $\left[V_{0}, V_{1}, V_{2}, V_{3}, V_{4}\right]$ is a subcomponent decomposition of $N$ and $\left[V_{0}^{\prime}, V_{1}, V_{2}, V_{3}, V_{4}\right]$ is a subcomponent decomposition $\theta(N)$. In addition, $N\left[V_{i}\right]$ is isomorphic to $\theta(N)\left[V_{i}\right]$ for all $0 \leq i \leq 4$. Since $N$ is level-1, Theorem 1 implies that $N\left[V_{i}\right]$ is level-1 for all $0 \leq i \leq 4$, and hence $\theta(N)\left[V_{i}\right]$ is also level-1 for all $0 \leq i \leq 4$. Employing Theorem 1 again, it follows that $\theta(N)$ is level-1. Finally, since $t\left(\widetilde{N}\left[V_{0}\right]\right)=t\left(\widetilde{\theta(N)}\left[V_{0}\right]\right)=2$, Eq. (1) in Theorem 1 implies $b(\theta(N))=b(N)$ and $t(\theta(N))=t(N)$.

Case II: $\theta$ is of type II. By interchanging the roles of $u$ and $v$ if necessary, we may assume without loss of generality that $u$ is contained in a cycle $H$ and that $v$ is a tree vertex. Let $A$ and $B$ denote the subcomponents of $N$ incident with $u_{1}$ and $u_{2}$, respectively, and let $C$ and $D$ denote the subcomponents of $N$ incident with $v$ that do not contain $u$ (see Fig. 6(i)). Then $\theta$ incorporates subcomponents $C$ and $D$ into the cycle containing $u$ and results in the network depicted in Fig. 6(ii) or in Fig. 6(iii). Without loss of generality, we may assume that $\theta(N)$ is as given in Fig. 6(ii) as the other case can be established in a similar manner. Since $N\left[V_{0}\right]$ and $\theta(N)\left[V_{0}^{\prime}\right]$ are both connected, $\left[V_{0}, V_{1}, V_{2}, V_{3}, V_{4}\right]$ and $\left[V_{0}^{\prime}, V_{1}, V_{2}, V_{3}, V_{4}\right]$ are subcomponent decompositions of $N$ and $\theta(N)$, respectively. In addition, $N\left[V_{i}\right]$ is isomorphic to $\theta(N)\left[V_{i}\right]$ for $1 \leq i \leq 4$. Since $\theta(N)\left[V_{0}^{\prime}\right]$ is obtained from $N\left[V_{0}\right]$ by (i) deleting the edge $\{u, v\}$ and the resulting isolated vertex $v$, and (ii) inserting a vertex into the cycle $H$, it follows that both $N\left[V_{0}\right]$ and $\theta(N)\left[V_{0}^{\prime}\right]$ have the same level, that $b\left(\theta(N)\left[V_{0}\right]\right)=b\left(N\left[V_{0}\right]\right)$, and that $\left.t \widetilde{(\theta(N)}\left[V_{0}\right]\right)=t\left(\widetilde{N}\left[V_{0}\right]\right)-1$. By Theorem 1 it follows that $\theta(N)$ is level-1, $b(\theta(N))=b(N)$, and $t(\theta(N))=t(N)-1$. 
Case III: $\theta$ is of type III. Let $H$ be the cycle in $N$ that contains both $u$ and $v$. Denote by $A$ the subcomponent of $N$ incident with $u_{1}$, by $B$ the subcomponent incident with $u$, by $C$ the subcomponent incident with $v$, and by $D$ the subcomponent incident with $v_{2}$ (see Fig. 7(i)). Since there are two subtypes of type III NNI operations, we divide our argument into the following two subcases.

The first subcase is that $\theta$ is type III-A. Then $\theta(N)$ is obtained from $N$ by swapping the relative positions of subcomponents $B$ and $C$, resulting in the network depicted in Fig. 7(ii). Hence in this subcase $\left[V_{0}, V_{1}, V_{2}, V_{3}, V_{4}\right]$ and $\left[V_{0}^{\prime}, V_{1}, V_{2}, V_{3}, V_{4}\right]$ are subcomponent decompositions of $N$ and $\theta(N)$, respectively. In addition, $N\left[V_{i}\right]$ is isomorphic to $\theta(N)\left[V_{i}\right]$ for all $0 \leq i \leq 4$. By Theorem 1 , the lemma follows using an argument similar to that in Case $\mathbf{I}$.

The second subcase is that $\theta$ is type III-B. Then $\theta(N)$ is obtained from $N$ by combining $B$ and $C$ into one subcomponent and shortening $H$ by one edge, that is, $\theta(N)$ is isomorphic to the network depicted in Fig. 7(iii). Since $N\left[V_{0}\right]$ and $\theta(N)\left[V_{0}^{\prime}\right]$ are both connected, $\left[V_{0}, V_{1}, V_{2}, V_{3}, V_{4}\right]$ and $\left[V_{0}^{\prime}, V_{1}, V_{2}, V_{3}, V_{4}\right]$ are subcomponent decompositions of $N$ and $\theta(N)$, respectively. In addition, $N\left[V_{i}\right]$ is isomorphic to $\theta(N)\left[V_{i}\right]$ for all $1 \leq i \leq 4$. Since $\theta(N)\left[V_{0}^{\prime}\right]$ is obtained from $N\left[V_{0}\right]$ by first suppressing $u$ and then attaching an edge to $v$, it follows that both $N\left[V_{0}\right]$ and $\theta(N)\left[V_{0}^{\prime}\right]$ have the same level, that $b\left(\theta(N)\left[V_{0}\right]\right)=b\left(N\left[V_{0}\right]\right)$, and that $\left.t \widetilde{(\theta(N)}\left[V_{0}\right]\right)=t\left(\widetilde{N}\left[V_{0}\right]\right)+1$. By Theorem 1, we obtain that $\theta(N)$ is level-1, that $b(\theta(N))=b(N)$, and that $t(\theta(N))=t(N)+1$.

A classical result by Robinson (1971) (see, also, Semple and Steel, 2003, Proposition 2.6.1) shows that any phylogenetic tree on $X$ can be converted into any other phylogenetic tree on $X$ by a sequence of NNI operations on such trees. We now show that an analogous result holds for LST operations on level-1 networks.

Theorem 2 Let $N$ and $N^{\prime}$ be two level-1 networks on $X$. Then $N^{\prime}$ can be obtained from $N$ by a sequence of LST operations.

Proof Note first that LST operations are invertible by Lemma 3 and the fact that TC operations are invertible. Since NNI operations can be used to convert any phylogenetic tree on $X$ into any other such tree, it suffices to show that if $N$ is a level-1 network on $X$ then there exists a sequence of LST operations converting $N$ into a phylogenetic tree on $X$.

We proceed by induction on the number $\beta(N)$ of vertices contained in the cycles of $N$. Since $\beta(N)=0$ if and only if $N$ is a phylogenetic tree, the base case $\beta(N)=0$ is trivial. For the induction step, assume that the required statement holds for all level-1 networks $M$ with $\beta(M)<m$ holding for some $m>0$ and that $\beta(N)=m$. Then $N$ must contain a cycle $C$. Consider an edge $e$ in $C$. Note that, by Lemma 2, $e$ is neither a chord nor a link of $N$. Let $N^{\prime}$ be a network obtained form $N$ by performing a type III-B NNI operation on $e$ if $C$ contains more than three vertices and a TC-shrink of $C$ otherwise. Then, in both cases, $N^{\prime}$ is obtained from $N$ by one LST operation and $\beta\left(N^{\prime}\right)<\beta(N)=m$. The theorem now follows by induction.

\section{LST neighborhoods of unrooted level-1 networks}

In this section, we consider the size of neighborhoods of level-1 networks under LST operations. Given a phylogenetic network on $X$, we let $\Gamma_{\mathrm{LST}}(N)$ denote the LST neighborhood of $N$, that is the set of all networks on $X$ that can be obtained from $N$ by a single LST operation. Note that for any phylogenetic tree $T$ on $n$ leaves the size of the neighborhood of $T$ using only NNI operations is $2 n-6$ (Robinson, 1971), which is independent of the topology of $T$.

Denoting for a phylogenetic network $N$ the number of links in $N$ by $l(N)$, we next establish the following result. 
Theorem 3 Let $N$ be a level-1 network with $n \geq 3$ leaves. Then

$$
\left|\Gamma_{\mathrm{LST}}(N)\right|=2 n-6+6 b(N)-5 b_{3}(N)-2 b_{4}(N)-2 l(N)+t(N) .
$$

To prove Theorem 3, we use two lemmas concerning LST operations. The first one (Lemma 5) concerns TC operations. Since its proof is straight-forward, we omit it.

Lemma 5 Suppose that $N$ is a level-1 network. Then the following holds.

(i) If $u$ and $u^{\prime}$ are two distinct tree vertices in $N$, then the network obtained from $N$ by a TC-pop on $u$ is distinct from that obtained from $N$ by a TC-pop on $u^{\prime}$.

(ii) If $C$ and $C^{\prime}$ are two distinct 3-cycles in $N$, then the network obtained from $N$ by a TC-shrink of $C$ is distinct from that obtained by a TC-shrink of $C^{\prime}$.

We next consider NNI operations. Given a phylogenetic network $N$ on $X$ and an interior edge $e$ of $N$. Then we denote by $\Gamma_{e}(N)$ the set of networks that can be obtained from $N$ by one NNI operation on $e$. Note that if $N$ is a tree then $\left|\Gamma_{e}(N)\right|=2$ and $\Gamma_{e}(N) \cap \Gamma_{e^{\prime}}(N)=\emptyset$ holds for any edge $e^{\prime}$ in $N$ distinct from $e$. Since $N$ has $n-3$ interior edges in that case, Robinson's aforementioned formula for the size of the NNI neighbourhood of phylogenetic trees follows, as also stipulated by Theorem 3. For level-1 networks that are not also phylogenetic trees, the situation is more complicated.

Lemma 6 Suppose that $N$ is a level-1 network on $X$, and that $e$ is an interior edge of $N$ that is neither a link nor contained in a 3-cycle of $N$. Then the following holds.

(i) $\left|\Gamma_{e}(N)\right|=2$.

(ii) Suppose that $e^{\prime}$ is an interior edge of $N$ that is distinct from e and is neither a link nor contained in a 3-cycle of $N$. If $e$ and $e^{\prime}$ are contained in the same 4-cycle of $N$ and do not share a vertex then $\left|\Gamma_{e}(N) \cap \Gamma_{e^{\prime}}(N)\right|=1$; otherwise $\left|\Gamma_{e}(N) \cap \Gamma_{e^{\prime}}(N)\right|=0$.

In particular, if $K$ is a 4-cycle in $N$, then there are precisely six non-isomorphic level-1 networks that result from performing one NNI operation on the edges in $K$.

Proof Let $e=\{u, v\}(u, v \in V(N))$ be an interior edge of $N$ that is neither a link nor contained in a 3-cycle of $N$. Then, by Lemma 2(i), $e$ is not a chord of $N$. Thus, $e$ is the axis of some NNI operations. Let $u_{i}\left(\right.$ resp. $\left.v_{i}\right), i=1,2$, be the two vertices adjacent to $u$ (resp. $v$ ) that are not in $e$. Then $u_{1}, u_{2}, v_{1}, v_{2}$ are four distinct vertices because $e$ is not contained in any 3-cycle. Put $f_{i}=\left\{u, u_{i}\right\}$ and $h_{i}=\left\{v, v_{i}\right\}$ for $i=1,2$. Consider the following eight triples: $\theta_{i}=\left(f_{i}, e, h_{i}\right)$ and $\theta_{i+2}=\left(h_{i}, e, f_{i}\right)$ for $i \in\{1,2\}, \theta_{5}=\left(f_{1}, e, h_{2}\right), \theta_{6}=\left(f_{2}, e, h_{1}\right), \theta_{7}=\left(h_{2}, e, f_{1}\right)$, and $\theta_{8}=\left(h_{1}, e, f_{2}\right)$. Note that an operation $\theta \in \Sigma(N)$ has axis $e$ if and only if $\theta \in\left\{\theta_{1}, \ldots, \theta_{8}\right\}$.

(i) To see that $\left|\Gamma_{e}(N)\right|=2$ holds, we distinguish between the following three cases.

Case i-1: Both $u$ and $v$ are tree vertices. Then $\theta_{i}$ is a type I NNI operation for $1 \leq i \leq 8$ (see Fig. 5(i) for an illustration). Since the networks in $\left\{\theta_{1}(N), \ldots, \theta_{4}(N)\right\}$ are pairwise isomorphic and the networks in $\left\{\theta_{5}(N), \ldots, \theta_{8}(N)\right\}$ are also pairwise isomorphic, it suffices to show that $\theta_{1}(N)$ is not isomorphic to $\theta_{5}(N)$. Denote the two subcomponents of $N$ incident with $u$ that do not contain $v$ by $A$ and $B$ and the two subcomponents incident with $v$ that do not contain $u$ by $C$ and $D$ (see Fig. 5(i)). Choose leaves $a \in A, b \in B, c \in C, d \in D$. Since $\theta_{1}(N)$ and $\theta_{5}(N)$ are given as in Fig. 5(iii) and Fig. 5(ii), respectively, the quartet $a c \mid b d$ is contained in $\mathscr{Q}\left(\theta_{1}(N)\right)$ but not in $\mathscr{Q}\left(\theta_{5}(N)\right)$. Thus, $\theta_{1}(N)$ is not isomorphic to $\theta_{5}(N)$, as required. 
Case i-2: Precisely one of $u$ and $v$ is a tree vertex. Swapping the roles of $u$ and $v$ if necessary, we may assume without loss of generality that $u$ is contained in a cycle of $N$ and $v$ is a tree vertex. Then $\theta_{i}$ is a type II NNI operation for $1 \leq i \leq 8$ (see Fig. 6(i) for an illustration). The case now follows using an argument similar to the one for Case i-1.

Case i-3: Both $\{u, v\}$ are contained in the same cycle (see Fig. 7(i) for an illustration). Then, for all $1 \leq i \leq 4$, the operation $\theta_{i}$ is type III-A and $\theta_{i}(N)$ is depicted in Fig. 7(ii). On the other hand, for $5 \leq i \leq 8$, the operation $\theta_{i}$ is of type III-B and $\theta_{i}(N)$ is depicted in Fig. 7(iii). Since, by Lemma 4, $t\left(\theta_{1}(N)\right) \neq t\left(\theta_{5}(N)\right)$ it follows that $\theta_{1}(N)$ is not isomorphic to $\theta_{5}(N)$. Combined with the fact that the networks in $\left\{\theta_{1}(N), \ldots, \theta_{4}(N)\right\}$ and also the networks in $\left\{\theta_{5}(N), \ldots, \theta_{8}(N)\right\}$ are pairwise isomorphic, we obtain that $\Gamma_{e}(N)=\left\{\theta_{1}(N), \theta_{5}(N)\right\}$, as required.

(ii) Let $u^{\prime}, v^{\prime} \in V(N)$ such that $e^{\prime}=\left\{u^{\prime}, v^{\prime}\right\}$ is an interior edge of $N$ that is distinct from $e$ and is neither a link nor contained in a 3-cycle of $N$. Then, by Lemma $2, e^{\prime}$ is not a chord of $N$. Thus, $e^{\prime}$ is the axis of some NNI operation on $N$. Without loss of generality, we may assume that the number of tree vertices in $\{u, v\}$ is greater than or equal to that in $\left\{u^{\prime}, v^{\prime}\right\}$. We distinguish between the following three cases.

Case ii-1: Both $u$ and $v$ are tree vertices. Let $A, B, C$, and $D$ denote the subcomponents of $N$ as in Case i-1 and choose leaves $a, b, c$, and $d$ as in that case. Then the quartet $a b \mid c d$ is not consistent with any network contained in $\Gamma_{e}(N)$. By swapping $u$ and $v$ if necessary, we may assume that $v$ is not contained in any path between $u$ and a vertex in $e^{\prime}$. Let $\theta^{\prime}$ be an arbitrary NNI operation on $e^{\prime}$. Then $v$ is a vertex in $\theta^{\prime}(N)$, and $C$ and $D$ are two subcomponents incident with $v$ in $\theta^{\prime}(N)$. Hence the quartet $a b \mid c d$ is consistent with $\theta^{\prime}(N)$, and, thus, is consistent with every network in $\Gamma_{e^{\prime}}(N)$. Hence, $\Gamma_{e}(N) \cap \Gamma_{e^{\prime}}(N)=\emptyset$, as required.

Case ii-2: Precisely one of $u$ and $v$ is a tree vertex. Without loss of generality, we may assume that $u$ is contained in a cycle of $N$ and that $v$ is a tree vertex. Let $A$ and $B$ denote subcomponents of $N$ incident with $u_{1}$ and $u_{2}$, respectively, and let $C$ and $D$ denote subcomponents of $N$ incident with $v$ that do not contain $u$. (see Fig. 6(i) for an illustration). Choose four leaves $a, b, c$, and $d$ as in Case i-1. Let $\mathscr{Q}=\{a b|c d, a c| b d, a d \mid b c\}$. Then $\left|\mathscr{Q}\left(N^{*}\right) \cap \mathscr{Q}\right|=2$ for all $N^{*} \in \Gamma_{e}(N)$. For instance, the network $N^{*}$ as illustrated in Fig. 6(ii) contains precisely two quartets in $\mathscr{Q}$ i. e. $a b \mid c d$ and $a d \mid b c$.

Choose an arbitrary network $N^{\prime} \in \Gamma_{e^{\prime}}(N)$ and let $\theta^{\prime}$ be the NNI operation on $e^{\prime}$ so that $N^{\prime}=\theta^{\prime}(N)$. It suffices to show that $N^{\prime} \notin \Gamma_{e}(N)$. To this end, we consider two subcases. If $u$ is incident with $e^{\prime}$ then $\theta^{\prime}$ is type III because $e^{\prime} \in$ $\left\{\left\{u, u_{1}\right\},\left\{u, u_{2}\right\}\right\}$. Noting that every NNI operation on $e$ is type II, Lemma 4 implies $t\left(N^{*}\right) \neq t\left(N^{\prime}\right)$ for all $N^{*} \in \Gamma_{e}(N)$. Hence $N^{\prime} \notin \Gamma_{e}(N)$ in this subcase. If $u$ is not incident with $e^{\prime}$ then $u$ is also a vertex in $N^{\prime}$ and is contained in every path between $x \in\{a, b\}$ and $y \in\{c, d\}$ in $N^{\prime}$. Thus, $\left|\mathscr{Q} \cap \mathscr{Q}\left(N^{\prime}\right)\right|=1$. Since, for all $N^{*} \in \Gamma_{e}(N)$, we must have $\left|\mathscr{Q}\left(N^{*}\right) \cap \mathscr{Q}\right|=2$ it follows that $N^{\prime} \notin \Gamma_{e}(N)$, as required.

Case ii-3: Both $u$ and $v$ are contained in some cycle $K$ of $N$. Since $|E(K)| \geq 4$ the vertices in $V(K)$ can be enumerated as $\left\{w_{1}, w_{2}, \ldots, w_{m}\right\}$ for some $m \geq 4$ such that $e_{i}:=\left\{w_{i}, w_{i+1}\right\}$ is an edge in $K$, for all $1 \leq i \leq m$. For simplicity, in the proof of this case we use the convention that the indices are calculated modulo $m$, that is, $w_{m+1}=w_{1}$. Moreover for all $1 \leq i \leq m$, let $H_{i}$ denote the subcomponent of $N$ that is incident with $w_{i}$ such that $V\left(H_{i}\right) \cap V(K)=\emptyset$ (see Fig. 8 for an illustration for the case $m=4$ ). For all $1 \leq i \leq m$, let $U_{i}$ denote the vertex set of $H_{i}$ and choose a leaf $x_{i} \in U_{i}$. Then $\left[V(K), U_{1}, \ldots, U_{m}\right]$ is a subcomponent decomposition of $N$. By an argument similar to the proof of Case i-3, we have, for all $1 \leq i \leq m$, that $\Gamma_{e_{i}}(N)=\left\{N_{i, A}, N_{i, B}\right\}$ where $N_{i, A}$ is obtained from $N$ by a type III-A NNI operation on $e_{i}$ that swaps the relative positions of subcomponents $H_{i}$ and $H_{i+1}$, and $N_{i, B}$ by a type III-B NNI operation on $e_{i}$ that combines the two subcomponents $H_{i}$ and $H_{i+1}$ into one subcomponent. Without loss of generality, we may assume that $e=e_{1}$, that is, 
$u=w_{1}$ and $v=w_{2}$. Since $\Gamma_{e}(N)=\Gamma_{e_{1}}(N)=\left\{N_{1, A}, N_{1, B}\right\}$, we have

$$
x_{m} x_{1} \mid x_{2} x_{3} \notin \mathscr{Q}\left(N^{*}\right) \quad \text { for all } N^{*} \in \Gamma_{e}(N) .
$$

Using the assumption that the number of tree vertices in $\{u, v\}$ is greater than or equal to that in $\left\{u^{\prime}, v^{\prime}\right\}$, it follows that $e^{\prime}$ is also contained in a cycle $K^{\prime}$ of $N$. Assume first that $K \neq K^{\prime}$. Then, by Theorem 1 , there exists some (necessarily unique) $1 \leq i \leq m$ such that $H_{i}$ contains $K^{\prime}$ and hence also contains $e^{\prime}$. Let $U_{i}^{c}=V(N) \backslash U_{i}$, and consider an arbitrary network $N^{\prime} \in \Gamma_{e^{\prime}}(N)$. Then $U_{i}^{c} \subseteq V\left(N^{\prime}\right)$ and $\left[U_{i}^{c}, V\left(N^{\prime}\right) \backslash U_{i}^{c}\right]$ is a subcomponent decomposition of $N^{\prime}$ such that $N\left[U_{i}^{c}\right]$ is isomorphic to $N^{\prime}\left[U_{i}^{c}\right]$. Thus, $x_{m} x_{1} \mid x_{2} x_{3} \in \mathscr{Q}\left(N^{\prime}\right)$. Combing with Eq. (2), we have $\Gamma_{e}(N) \cap \Gamma_{e^{\prime}}(N)=\emptyset$, as required for this subcase.

Assume for the remainder that $K=K^{\prime}$. Then there must exist some $2 \leq i \leq m$ such that $e^{\prime}=e_{i}$. We next analyse the networks $N_{1, A}, N_{1, B}, N_{i, A}$ and $N_{i, B}$.

We claim first that $N_{1, A}$ and $N_{1, B}$ are not isomorphic and also that $N_{i, A}$ and $N_{1, B}$ are not isomorphic. Indeed, by Lemma 4 we have $t\left(N_{1, A}\right) \neq t\left(N_{i, B}\right)$ and $t\left(N_{i, A}\right) \neq t\left(N_{1, B}\right)$, from which the claim follows.

Secondly, we claim that $N_{1, B}$ and $N_{i, B}$ are not isomorphic. Indeed, this follows by noting that in $N_{1, B}$ the two subcomponents $H_{1}$ and $H_{2}$ are incident with the same vertex whilst in $N_{i, B}$ the two vertices incident with $H_{1}$ and $H_{2}$ are distinct.

We conclude the proof of (ii) by claiming that $N_{1, A}$ and $N_{i, A}$ are isomorphic if and only if $m=4$ and $i=3$. To establish the 'if' direction, suppose that $m=4$ and $i=3$. Then $N_{1, A}$ and $N_{3, A}$ are as depicted in Figure 8(ii) and (iii), respectively. Clearly, $N_{1, A}$ and $N_{3, A}$ are isomorphic.

To establish the 'only if' direction, we use contraposition. To this end, suppose first that $m>4$; then we need to show that $N_{1, A}$ and $N_{i, A}$ are not isomorphic. Clearly, $m>4$ implies $\left\{x_{i}, x_{i+1}\right\} \neq\left\{x_{3}, x_{m}\right\}$. Since, when restricting to $\left\{x_{1}, \ldots, x_{m}\right\}$, only the relative position of $x_{i}$ and $x_{i+1}$ in $N_{i, A}$ are swapped, we have $x_{m} x_{1} \mid x_{2} x_{3} \in \mathscr{Q}\left(N_{i, A}\right)$. Therefore $N_{1, A}$ and $N_{i, A}$ are not isomorphic as $x_{m} x_{1} \mid x_{2} x_{3} \notin \mathscr{Q}\left(N_{i, A}\right)$, in view of Eq. (2). Next, suppose that $m=4$ and $i \neq 3$. Then $i \in\{2,4\}$, and an argument similar to the one in the case $m>4$ implies that $x_{4} x_{1} \mid x_{2} x_{3} \in \mathscr{Q}\left(N_{2, A}\right)$ and $x_{4} x_{1} \mid x_{2} x_{3} \in \mathscr{Q}\left(N_{4, A}\right)$. Since $x_{4} x_{1} \mid x_{2} x_{3} \notin \mathscr{Q}\left(N_{1, A}\right)$, it follows that neither $N_{2, A}$ nor $N_{4, A}$ is isomorphic to $N_{1, A}$, which completes the proof of the last claim.

Since $\Gamma_{e}(N)=\left\{N_{1, A}, N_{1, B}\right\}$ and $\Gamma_{e^{\prime}}(N)=\left\{N_{i, A}, N_{i, B}\right\}$, by the above three claims it follows that $\left|\Gamma_{e}(N) \cap \Gamma_{e^{\prime}}(N)\right|$ is 1 if $m=4$ and $e^{\prime}=e_{3}$, and 0 otherwise. Since $e=e_{1}$, the condition that $m=4$ and $e^{\prime}=e_{3}$ is equivalent to that $e$ and $e^{\prime}$ are two disjoint edges in the same 4-cycle. This concludes the proof of Case ii-3 and thus the proof of (ii).

It remains to establish the last statement of the theorem. Assume that $K$ is a 4-cycle in $N$. For simplicity, we use the same notation as in the proof of Case ii-3 with the additional assumption that $m=4$ (see Fig. 8 for an illustration). By an argument similar to the proof of Case ii-3, we have

$$
\left|\Gamma_{e_{i}}(N) \cap \Gamma_{e_{j}}(N)\right|= \begin{cases}1 & \text { if }(i, j)=(1,3) \text { or }(i, j)=(2,4), \\ 0 & \text { otherwise }\end{cases}
$$

for $1 \leq i<j \leq 4$, and hence also $\left|\Gamma_{e_{i}}(N) \cap \Gamma_{e_{j}}(N) \cap \Gamma_{e_{t}}(N)\right|=0$ for $1 \leq i<j<t \leq 4$. Together with the principle of inclusion and exclusion, this yields

$$
\left|\bigcup_{1 \leq i \leq 4} \Gamma_{e_{i}}(N)\right|=\sum_{i=1}^{4}\left|\Gamma_{e_{i}}(N)\right|-\sum_{1 \leq i<j \leq 4}\left|\Gamma_{e_{i}}(N) \cap \Gamma_{e_{i}}(N)\right|=6,
$$


where for the second equality we use the fact that $\left|\Gamma_{e_{i}}(N)\right|=2$ holds for all $1 \leq i \leq 4$ in view of Lemma 6 (i). Thus, there are precisely six non-isomorphic level-1 networks that result from performing one NNI operation on edges in $K$, as required.

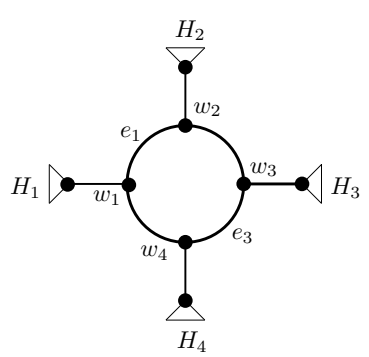

(i)

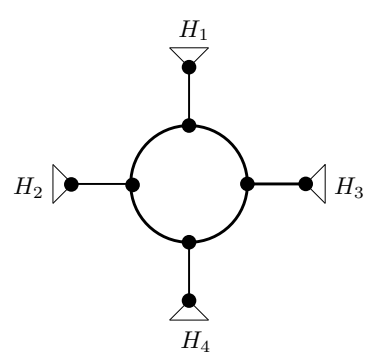

(ii)



(iii)

Fig. 8 An illustration for a step in the proof of Lemma 6 (ii). Here the network $N$ in (i) contains a cycle $K$ with vertices $w_{1}, w_{2}, w_{3}, w_{4}$. The networks in (ii) and (iii) are $N_{1, A}$ and $N_{3, A}$, respectively.

With Lemmas 5 and 6 , we are now in a position to present the proof of Theorem 3 .

\section{Proof of Theorem 3}

Let $\Gamma_{0}(N)$ be the set of networks obtained from $N$ by performing one TC-shrink. Then $\left|\Gamma_{0}(N)\right|=b_{3}(N)$ by Lemma 5 . Moreover, denoting by $\Gamma_{2}(N)$ the set of networks obtained from $N$ by a TC-pop, the same lemma implies $\left|\Gamma_{2}(N)\right|=t(N)$.

Let $\Gamma_{1}(N)$ be the set of networks obtained from $N$ by performing one NNI operation. To count the number of networks in $\Gamma_{1}(N)$, note first that Lemma 1 implies that the number of interior edges in $N$ is $n-3+3 b(N)$. Since the axis of an NNI operation is an interior edges that is neither a link nor contained in a 3-cycle, the number of interior edges in $N$ on which an NNI operation can be performed is $n-3+3 b(N)-3 b_{3}(N)-l(N)$. Hence, by Lemma 6 we have

$$
\left|\Gamma_{1}(N)\right|=2\left[n-3+3 b(N)-3 b_{3}(N)-l(N)-4 b_{4}(N)\right]+6 b_{4}(N)
$$

To complete the proof, let $i \in\{0,1,2\}$. Then for each $N^{\prime} \in \Gamma_{i}(N)$ we have $b\left(N^{\prime}\right)=b(N)-1+i$ in view of Lemma 4 . This implies $\Gamma_{i}(N) \cap \Gamma_{j}(N)=\emptyset$ for $0 \leq i<j \leq 2$, and hence we have

$$
\begin{aligned}
\left|\Gamma_{\mathrm{LST}}(N)\right| & =\left|\Gamma_{0}(N)\right|+\left|\Gamma_{1}(N)\right|+\left|\Gamma_{2}(N)\right| \\
& =2 n-6+6 b(N)-5 b_{3}(N)-2 b_{4}(N)-2 l(N)+t(N),
\end{aligned}
$$

as required.

In view of Theorem 3, it is of interest to understand which level-1 networks have the smallest and largest neighborhoods. We next characterize those having the smallest possible neighborhoods. To this end, we call a network $N$ saturated if every interior vertex of $N$ is contained in a 3-cycle.

Theorem 4 Suppose that $N$ is a level-1 network with $n \geq 4$ leaves. Then $\left|\Gamma_{\mathrm{LST}}(N)\right| \geq n-2$, and this bound is attained if and only if $N$ is saturated.

Proof For clarity of exposition, we remark that all networks considered are assumed to be level-1. In addition, put

$$
\varphi(N)=\left|\Gamma_{\mathrm{LST}}(N)\right|-(2 n-6)=6 b(N)-5 b_{3}(N)-2 b_{4}(N)-2 l(N)+t(N)
$$


for every network $N$ with $n$ leaves. Informally, $\varphi(N)$ is the number of level-1 networks that are one LST operation away from $N$ minus the number of phylogenetic trees that are one LST operation away from a phylogenetic tree with $n$ leaves. Let $N_{0}$ denote a saturated network on $n \geq 4$ leaves. Then the fact that a phylogenetic tree on $n$ leaves has $n-2$ interior vertices and $n-3$ interior edges combined with the fact that every blob of $N_{0}$ is a 3-cycle implies that $b\left(N_{0}\right)=n-2=b_{3}\left(N_{0}\right), b_{4}\left(N_{0}\right)=t\left(N_{0}\right)=0, l\left(N_{0}\right)=n-3$. Hence, $\varphi\left(N_{0}\right)=4-n$ and, so, $\left|\Gamma_{\mathrm{LST}}\left(N_{0}\right)\right|=n-2$.

Let $N^{\prime}$ be a network on $n$ leaves so that $\varphi\left(N^{\prime}\right) \leq \varphi(N)$ holds for all networks $N$ on $n$ leaves. To complete the proof, it suffices to show that $N^{\prime}$ is saturated. To this end, we first show that $N^{\prime}$ does not contain any cycle with more than three vertices. Assume for contradiction that $N^{\prime}$ contains a cycle $K$ with $|V(K)| \geq 4$. Choose an edge $e$ in $K$ and let $N^{*}$ denote the network obtained from $N^{\prime}$ by a type III-B NNI operation on $e$, followed by a TC-pop at the newly created vertex $v$ (see Fig. 9 for an illustration). Note that if $|V(K)|>4$, then

$$
b\left(N^{*}\right)=b\left(N^{\prime}\right)+1, \quad t\left(N^{*}\right)=t\left(N^{\prime}\right), \quad b_{3}\left(N^{*}\right)=b_{3}\left(N^{\prime}\right)+1, \quad l\left(N^{*}\right)=l\left(N^{\prime}\right)+1,
$$

which implies $\varphi\left(N^{\prime}\right)-\varphi\left(N^{*}\right)=1+2\left(b_{4}\left(N^{*}\right)-b_{4}\left(N^{\prime}\right)\right)>0$ as $b_{4}\left(N^{*}\right)=b_{4}\left(N^{\prime}\right)$ also holds. If $K$ is a 4-cycle then a similar argument implies $\varphi\left(N^{\prime}\right)-\varphi\left(N^{*}\right)=4$. Hence, in both cases, it follows that $\varphi\left(N^{\prime}\right)>\varphi\left(N^{*}\right)$ in contradiction to the choice of $N^{\prime}$. Thus, any cycle in $N^{\prime}$ must be a 3-cycle. But then $b_{3}\left(N^{\prime}\right)+t\left(N^{\prime}\right)=n-2$, because applying one TC-shrink to every 3-cycle in $N^{\prime}$ results in a phylogenetic tree with $n$ leaves. Thus, $\varphi\left(N^{\prime}\right)=n-2-2 l\left(N^{\prime}\right)$ in view of $b_{4}\left(N^{\prime}\right)=0$ and $b\left(N^{\prime}\right)=b_{3}\left(N^{\prime}\right)$. In addition, by Lemma 2, $l\left(N^{\prime}\right)$ is bounded from above by the number of interior edges in $N^{\prime}$ minus $3 b\left(N^{\prime}\right)$ because each non-trivial blob in $N^{\prime}$ contains at least three edges. Together with Lemma 1 , this implies

$$
l\left(N^{\prime}\right) \leq n-3+3 b\left(N^{\prime}\right)-3 b\left(N^{\prime}\right)=n-3
$$

where the inequality becomes an equality if and only if $N^{\prime}$ is saturated. Therefore,

$$
\varphi\left(N^{\prime}\right)=n-2-2 l\left(N^{\prime}\right) \geq n-2-2(n-3)=4-n=\varphi\left(N_{0}\right)
$$

where the inequality becomes an equality if and only if $N^{\prime}$ is saturated. The choice of $N^{\prime}$ implies $\varphi\left(N^{\prime}\right)=\varphi\left(N_{0}\right)$, from which it follows that $N^{\prime}$ must be saturated.

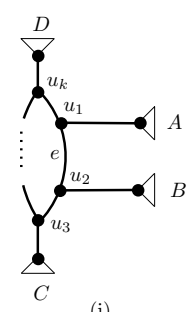

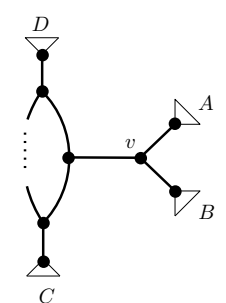

(ii)

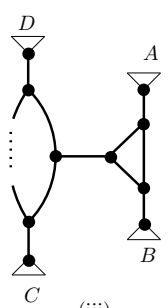

(iii)

Fig. 9 An illustration of the construction in the proof of Theorem 4. Here the network in (ii) is obtained from the network $N^{\prime}$ in (i) by one NNI operation on $e$, and the network $N^{*}$ in (iii) is obtained from the network in (ii) by a TC-pop on $v$. The vertices $u_{1}, u_{2}, \ldots, u_{k}$ denote the vertices of $K$.

Remark 1 It would be interesting to characterize those level-1 networks with largest LST neighborhoods. Let $N$ be such a network. Then, using an argument similar to the proof of Theorem 4 it is possible to show that we can assume that each cycle in $N$ has length 4 or 5 . However, we have not been able to characterize the possible structures for $N$. 


\section{LST metrics on level-1 networks}

When restricted to phylogenetic trees, NNI operations induce a metric on the set of all such trees, where the metric between two trees is the minimum number of such operations required to convert one into the other (see e.g. Semple and Steel $\left(2003\right.$, p.33)). In a similar vein, we define the LST metric $d_{\text {LST }}$ between any pair of level-1 networks to be the minimum number of LST operations required to convert one into the other. This is a metric on level-1 networks on a fixed leaf set since it corresponds to the shortest path metric on the graph whose vertices are level-1 networks, and whose edges are pairs of such networks that differ by one LST operation, and implied by Theorem 2, this graph is connected (see, e.g. Fig. 1 for an example).

Note that if we are given two level-1 networks on $X$ each with exactly one cycle of length $n \geq 3$, we could move between these two networks by just swapping adjacent pairs of leaves using only type-III B NNI operations. This scenario is related to the edit distance between circular permutations or genomes (see e.g. Chen and Skiena, 1996) and we can therefore think of $d_{\text {LST }}$ as a generalization of circular permutation edit distance.

It is an interesting problem to understand the diameter of the LST metric, that is the largest possible value of $d_{\mathrm{LST}}\left(N, N^{\prime}\right)$ over all pairs of level-1 networks $N, N^{\prime}$ with leaf set $X$. Note that the diameter of the NNI metric on phylogenetic trees on $X$ is bounded above and below by functions that grow at the rate of $n \log (n)$ (Li et al, 1996). Using this fact we now give an upper bound on the diameter of $d_{\mathrm{LST}}$.

Theorem 5 Let $\Delta(n)$ be the diameter of $d_{\mathrm{LST}}$ on the set of level-1 networks on $X$. Then $\Delta(n) \in \Omega(n)$ and $\Delta(n) \in$ $O(n \log (n))$.

To prove this result, we use the following lemma.

Lemma 7 Let $N$ be a level-1 network on $X$. Then

$$
\min \left\{d_{\mathrm{LST}}(N, T): T \text { is a phylogenetic tree on } X\right\}=n-2-t(N) .
$$

Proof Let $N$ be as in the statement of the lemma and set $m=b(N)$. Let $H_{1}, \cdots, H_{m}$ denote the cycles in $N$. Since each interior vertex in $N$ is either a tree vertex or contained in a cycle, Lemma 1 implies $\sum_{i=1}^{m}\left|V\left(H_{i}\right)\right|=n-2+2 b(N)-t(N)$.

To convert $N$ into a phylogenetic tree on $X$ by a sequence of LST operations, it is necessary for each cycle $H_{i}$ $(1 \leq i \leq m)$ to be removed. This can be achieved by repeatedly performing a type III-B NNI operation on an edge in $H_{i}$ that reduces the length of $H_{i}$ by one if $\left|V\left(H_{i}\right)\right| \geq 4$ and then performing a TC-shrink when a cycle of length 3 is obtained. In particular, any cycle $H_{i}$ in $N$ can be removed by performing a sequence of $\left|V\left(H_{i}\right)\right|-2$ LST operations. Moreover, since all other LST operations do not affect the length of the cycles in $N$, the cycle $H_{i}$ cannot be removed by performing less than $\left|V\left(H_{i}\right)\right|-2$ LST operations. Thus, the minimum number of LST operations required to convert $N$ into a phylogenetic tree is given by

$$
\sum_{i=1}^{m}\left(\left|V\left(H_{i}\right)\right|-2\right)=-2 b(N)+\sum_{i=1}^{m}\left|V\left(H_{i}\right)\right|=n-2-t(N),
$$

as required.

Proof of Theorem 5: The fact that $\Delta(n) \in O(n \log (n))$ holds follows immediately from Lemma 7, and the fact that the NNI metric between any pair of phylogenetic trees on $X$ is bounded above by a function that grows at rate $n \log (n)$. 
To see that $\Delta(n) \in \Omega(n)$ holds, let $T$ be any phylogenetic tree on $X$ and let $N$ be the level-1 network obtained by applying a TC-pop operation to each of the interior vertices of $T$. By Lemma 7, it follows that $d_{\mathrm{LST}}(N, T)=n-2$.

It would be interesting to obtain sharp bounds for the diameter of $d_{\mathrm{LST}}$. Note that it is known that computing the NNI metric between any two phylogenetic trees is NP-complete (DasGupta et al, 1997), and so it would be of some interest to decide whether or not this also holds for the LST metric between two networks.

\section{LST operations on rooted level-1 networks}

In this section, we extend our study of LST operations to rooted level-1 networks. To this end, we begin by presenting some additional notation and terminology. Given a directed graph $G$, let $A(G)$ denote the set of its arcs and let $U(G)$ be the underlying graph of $G$. We denote the tail of an arc $a$ by $t_{a}$ and the head of $a$ by $h_{a}$, and use the convention that $U(a)=\left\{t_{a}, h_{a}\right\}$. Recall that a rooted (phylogenetic) network $N$ on $X$ is a directed graph that does not contain any directed cycles, and in which exactly one vertex has indegree 0 and outdegree 2 (the root, usually denoted by $\rho_{N}$ ) and all other vertices have either indegree 1 and outdegree 2, indegree 2 and outdegree 1 (hybrid vertices), or indegree 1 and outdegree 0 (the leaves). As with unrooted networks, the set of leaves of $N$ is equal to $X$ and $U(N)$ is connected. A subgraph $C$ of $N$ whose underlying graph in $U(N)$ is a cycle is called a gall of $N$, and a gall is called trivial if it has three vertices. A rooted phylogenetic network $N$ called is level-1 if and only if $U(N)$ is level-1, that is, each pair of distinct galls contained in $N$ is vertex disjoint.

To simplify the definition of our rooted version of the NNI operation, we follow the approach in (Bordewich and Semple, 2005; Baroni et al, 2005) (where a rooted version of the subtree prune and redraft operation on phylogenetic trees is defined) by introducing a family of directed graphs that is closely related to rooted networks: A planted network on $X$ is a directed graph with a unique vertex $\pi$ that has indegree 0 such that $\pi$ has outdegree 1 , and removing $\pi$ and the arc directed from $\pi$ results in a rooted network on $X$. Suppose $N$ is a rooted network on $X$. Then the directed graph $P(N)$ obtained from $N$ by adding a new vertex $\pi$ and an incoming $\operatorname{arc}\left(\pi, \rho_{N}\right)$ is a planted network on $X$ and will be referred to as the planting of $N$. For ease of terminology we call $\mathscr{N}=P(N)$ a planted level-1 network (on $X$ ) when $N$ is a rooted level-1 network (on $X$ ). Note that the mapping that associates a rooted level-1 network $N$ to $P(N)$ is a bijection from the set of rooted level-1 networks on $X$ to the set of planted level-1 networks on $X$.

For $\mathscr{N}=P(N)$, let $\Sigma^{r}(\mathscr{N})$ denote the set of all ordered triplets $\theta:=\left(a_{1}, a, a_{2}\right)$ of three distinct $\operatorname{arcs}$ in $A(\mathscr{N})$ such that (A1) $a a_{1}$ is a directed path (that is, $h_{a}=t_{a_{1}}$ ), (A2) $U\left(a_{2}\right) \cap U(a)=\left\{t_{a}\right\}$ and $U\left(a_{1}\right) \cap U\left(a_{2}\right)=\emptyset$, (A3) $a$ is not contained in a trivial gall, $t_{a}$ and $h_{a}$ are not contained in two distinct galls, and neither $t_{a}$ nor $h_{a}$ is a hybrid vertex. Here the $\operatorname{arc} a$ is called the axis of $\theta$. Note that, conditions (A1)-(A3) imply that $a$ is an interior arc of $\mathscr{N}$ and that $\left\{t_{a}, h_{a}\right\}$ is neither a link nor contained in a 3-cycle of $U(\mathscr{N})$. As in the unrooted case, we associate to a triple $\theta=\left(a_{1}, a, a_{2}\right)$ in $\Sigma^{r}(\mathscr{N})$ a planted network $\theta(\mathscr{N})$ with leaf set $X$ obtained as follows: Subdivide $a_{1}$ and $a_{2}$ by new vertices $u^{\prime}$ and $v^{\prime}$, respectively; add the arc $\left(v^{\prime}, u^{\prime}\right)$; delete the arc $\left(h_{a}, u^{\prime}\right)$, and suppress resulting degree two vertices (see Fig. 10 for an illustration). We call this operation an $r$ NNI operation on $\mathscr{N}$. With this in mind, we obtain the following result.

Lemma 8 Suppose $N$ is a rooted level-1 network on $X$ and $a_{1}$, a, and $a_{2}$ are arcs of its planting $\mathscr{N}=P(N)$ such that $\theta=\left(a_{1}, a, a_{2}\right)$ is an $r \mathrm{NNI}$ operation on $\mathscr{N}$. Then $U(\mathscr{N})$ is a level-1 network on $X \cup\{\pi\}$, the triple $\theta^{*}=$ $\left(U\left(a_{1}\right), U(a), U\left(a_{2}\right)\right)$ is an NNI operation on $U(\mathscr{N})$, and $\theta^{*}(U(\mathscr{N}))=U(\theta(\mathscr{N}))$. In particular, $\theta(\mathscr{N})$ is a planted level-1 network on $X$. 



Fig. 10 An illustration of an rNNI operation: the network in (iii) is obtained from that in (i) by the rNNI operation $\left(a_{1}, a, a_{2}\right)$ with $a=(v, u)$. Here all arcs are directed away from the vertex $\pi$.

Proof Since $N$ is a rooted level-1 network on $X$, the graph $U(N)$ is connected and level-1. In addition, except for the vertex $\rho_{N}$ which has degree two and the vertices in the leaf set $X$, every vertex in $U(N)$ has degree three. Noting that $U(\mathscr{N})$ can be obtained from $U(N)$ by adding an edge between $\rho_{N}$ and a newly added leaf $\pi$, it follows that $U(\mathscr{N})$ is a level-1 network on $X \cup\{\pi\}$.

To see that $\theta^{*}$ is an NNI operation on $U(\mathscr{N})$, note that, by Lemma 2(i), $U(a)$ is not a chord in $U(\mathscr{N})$. In addition, $U\left(a_{1}\right), U(a), U\left(a_{2}\right)$ is a path in $U(\mathscr{N})$ and $U(a)$ is neither a link nor contained in a 3-cycle of $U(\mathscr{N})$. Thus, $\theta^{*}$ is an NNI operation on $U(\mathscr{N})$.

To see that $\theta^{*}(U(\mathscr{N}))=U(\theta(\mathscr{N}))$, note first that to obtain $\theta(\mathscr{N})$ from $\mathscr{N}$, we subdivide the arcs $a_{1}$ and $a_{2}$ in $\theta(\mathscr{N})$ by new vertices $u^{\prime}$ and $v^{\prime}$, respectively if and only if we subdivide the edges $U\left(a_{1}\right)$ and $U\left(a_{2}\right)$ in $U(\mathscr{N})$ by new vertices $u^{\prime}$ and $v^{\prime}$, respectively. Next, note that we add the $\operatorname{arc}\left(v^{\prime}, u^{\prime}\right)$ to $\theta(\mathscr{N})$ and delete $\left(h_{a}, u^{\prime}\right)$ if and only if we add the edge $\left\{v^{\prime}, u^{\prime}\right\}$ to $U(\mathscr{N})$ and delete the edge $\left\{u^{\prime}, u=h_{a}\right\}$. Moreover, note that a vertex in $U(\mathscr{N})$ has been rendered a degree two vertex by the above process if and only if it has been rendered a degree two vertex in $\theta(\mathscr{N})$. Thus, $\theta^{*}(U(\mathscr{N}))=U(\theta(\mathscr{N}))$.

Finally, by Lemma 4 it follows that $\theta^{*}(U(\mathscr{N}))$ is level-1 and hence $U(\theta(\mathscr{N}))$ is level-1. Therefore $\theta(\mathscr{N})$ is a planted level-1 network on $X$, as required.

Given two rooted level-1 networks $N$ and $N^{\prime}$ on $X$, we say $N^{\prime}$ can be obtained from $N$ by one $r$ NNI operation if $P\left(N^{\prime}\right)$ can be obtained from $P(N)$ by one rNNI operation $\theta$. In this case, we also write $N^{\prime}=\theta(N)$. We now show that, just as with unrooted level-1 networks, the rNNI operation can also be reversed.

Lemma 9 Suppose that $N$ is a rooted level-1 network on $X$ and let $\theta$ be an $r$ NNI operation on $N$. Then there exists an $r$ NNI operation $\theta^{\prime}$ on $\theta(N)$ such that $N=\theta^{\prime}(\theta(N))$.

Proof It suffices to consider the statement for the planted version $\mathscr{N}=P(N)$ of $N$. To this end, let $a$ be the axis of $\theta$ and put $u=h_{a}, v=t_{a}$. For simplicity, put $\mathscr{N}^{\prime}=\theta(\mathscr{N})$. Then by Lemma 8 it follows that $\mathscr{N}^{\prime}$ is a planted level-1 network. Since $\theta$ is an rNNI operation on $a$, there exist four distinct vertices $u_{1}, u_{2}, v_{1}, v_{2}$ in $V(\mathscr{N})-\{u, v\}$ such that $a_{1}=\left(u, u_{1}\right), a_{3}=\left(u, u_{2}\right), a_{4}=\left(v_{1}, v\right)$, and $a_{2}=\left(v, v_{2}\right)$ are four arcs in $\mathscr{N}$. Swapping $u_{1}$ and $u_{2}$ if necessary, we assume that $a_{1}$ is an arc contained in $\theta$. In addition, we may assume that $\theta=\left(a_{1}, a, a_{2}\right)$, as the case $\theta=\left(a_{1}, a, a_{4}\right)$ can be established in a similar manner. 
Let $v^{\prime}$ and $u^{\prime}$ be the vertices that divide $a_{2}$ and $a_{1}$ in the construction of $\mathscr{N}^{\prime}$. Then $a_{1}^{\prime}=\left(v^{\prime}, u_{1}\right), a^{\prime}=\left(v, v^{\prime}\right), a_{2}^{\prime}=$ $\left(v, u_{2}\right)$, and $a_{3}^{\prime}=\left(v^{\prime}, v_{2}\right)$ are $\operatorname{arcs}$ in $\mathscr{N}^{\prime}$, and we have

$$
V\left(\mathscr{N}^{\prime}\right)=(V(\mathscr{N})-\{u\}) \cup\left\{v^{\prime}\right\} \quad \text { and } \quad A\left(\mathscr{N}^{\prime}\right)=\left(A(\mathscr{N})-\left\{a_{1}, a_{2}, a_{3}, a\right\}\right) \cup\left\{a_{1}^{\prime}, a_{2}^{\prime}, a_{3}^{\prime}, a^{\prime}\right\} .
$$

To define $\theta^{\prime}$, note first that $v^{\prime}$ and $v$ are not contained in two distinct galls of $\mathscr{N}$ as this would imply none of the edges in $\left\{U(a), U\left(a_{1}\right), U\left(a_{2}\right), U\left(a_{3}\right), U\left(a_{4}\right)\right\}$ is a cut edge in $U(\mathscr{N})$, a contradiction to the fact that $N$ is a rooted level-1 network. Also $v$ and $v^{\prime}$ cannot be contained in a trivial gall of $\mathscr{N}^{\prime}$ because $\mathscr{N}$ neither has multiple arcs nor contains the $\operatorname{arc}\left(v, u_{1}\right)$. Together with the observation that, by construction, neither $v^{\prime}$ nor $v$ is a hybrid vertex in $\mathscr{N}^{\prime}$ and that Properties (A1) and (A2) are satisfied for the namesakes of $a_{1}^{\prime}, a^{\prime}$, and $a_{2}^{\prime}$ in their respective statements, it follows that the triple $\theta^{\prime}=\left(a_{1}^{\prime}, a^{\prime}, a_{2}^{\prime}\right)$ is an rNNI operation on $\mathscr{N}^{\prime}$. A straight forward check implies $\mathscr{N}=\theta^{\prime}\left(\mathscr{N}^{\prime}\right)$ and, so, the lemma follows.

Just as with the unrooted case we now define two more operations which concern trivial galls. Let $N$ denote a rooted level-1 network and let $v$ denote a branching vertex of $N$, that is, $v$ is neither a leaf nor a contained in a gall. Let $a$ and $a^{\prime}$ denote its two outgoing arcs and let $u$ and $u^{\prime}$ be two vertices that subdivide $a$ and $a^{\prime}$, respectively. Then we obtain a rooted level-1 network $N_{\left(a, a^{\prime}\right)}$ from $N$ by adding an $\operatorname{arc}\left(u, u^{\prime}\right)$ from $u$ to $u^{\prime}$ which renders $u^{\prime}$ a hybrid vertex of $N_{\left(a, a^{\prime}\right)}$. Thus $N_{\left(a, a^{\prime}\right)}$ has one more gall than $N$ and we say that $N_{\left(a, a^{\prime}\right)}$ is obtained from $N$ via a rooted TC-pop. Note that $N_{\left(a^{\prime}, a\right)}$ and $N_{\left(a, a^{\prime}\right)}$ are distinct rooted level-1 networks because the new hybrid vertex in $N_{\left(a^{\prime}, a\right)}$ is $u$.

Conversely, if $C$ is a trivial gall of $N$, then we obtain a rooted level-1 network $N^{\prime}$ from $N$ by first deleting an arc $a$ in $C$ for which $h_{a}$ is the hybrid vertex of $C$, and then suppressing resulting vertices with indegree one and outdegree one. In this case, we say that $N^{\prime}$ is obtained from $N$ by a rooted TC-shrink of $C$. Note that $N^{\prime}$ is a rooted level-1 network that has one gall less than $N$.

If $N$ and $N^{\prime}$ are two rooted level-1 networks on $X$ and $N^{\prime}$ can be obtained from $N$ by either a rooted TC-pop, a rooted TC-shrink, or an rNNI operation then we say that $N^{\prime}$ can be obtained from $N$ by a rooted local subnetwork transfer (rLST) operation. Since $N$ can be obtained from $N^{\prime}$ by a rooted TC-shrink if and only if $N^{\prime}$ can obtained from $N$ by a rooted TC-pop, Lemma 9 immediately implies that rooted LST operations are reversible.

Lemma 10 Suppose that $N$ and $N^{\prime}$ are two rooted level-1 networks on $X$. Then $N$ can be obtained from $N^{\prime}$ by one $r$ LST operation if and only if $N^{\prime}$ can be obtained from $N$ by one $r$ LST operation.

We next present the main result of this section which can be thought of as the analogue of Theorem 2 in that rLST operations as defined above are sufficient to transform a rooted level-1 network on $X$ into any other such network.

Theorem 6 Let $N$ and $N^{\prime}$ be two rooted level-1 networks on $X$. Then $N^{\prime}$ can be obtained from $N$ by a finite sequence of $r$ LST operations.

Proof Since rLST operations are invertible by Lemma 10 and rNNI operations can be used to convert any rooted phylogenetic tree into any other, it suffices to show that if $N$ is a rooted level-1 network on $X$, then there exists a sequence of rLST operations transforming $N$ into a rooted phylogenetic tree on $X$.

We proceed by induction on the number $\beta^{r}(N)$ of vertices in a rooted level-1 network $N$ that are contained in some gall. Since $\beta^{r}(N)=0$ if and only if $N$ is a rooted phylogenetic tree, the base case $\beta^{r}(N)=0$ is trivial. For the induction 
step, assume that the required statement holds for all rooted level-1 networks $N^{*}$ with $\beta^{r}\left(N^{*}\right)<m$ for some $m>0$, and that $\beta^{r}(N)=m$.

Since $\beta^{r}(N)>0$, there exists a gall $C$ in $N$. If $C$ is trivial then let $N^{\prime}$ denote the rooted level-1 network obtained from $N$ by performing a TC-shrink of $C$. Otherwise, put $\mathscr{N}=P(N)$ and let $a_{2}$ be the arc in $\mathscr{N}$ such that $h_{a_{2}}$ is contained in $C$ but $t_{a_{2}}$ is not, and let $a$ be an arc in $C$ such that $h_{a_{2}}=t_{a}$ and $h_{a}$ is not the hybrid vertex of $C$. In addition, let $a_{1}$ be the arc of $\mathscr{N}$ with $t_{a_{1}}=h_{a}$ and $h_{a_{1}}$ is not contained in $C$. Let $\mathscr{N}^{\prime}$ be the planted level-1 network obtained from $\mathscr{N}$ by applying the rNNI operation $\theta=\left(a_{1}, a, a_{2}\right)$, and let $N^{\prime}$ be the rooted level-1 network on $X$ obtained from $\mathscr{N}^{\prime}$ by removing the unique arc whose tail has indegree 0 . Then in both cases $N^{\prime}$ is obtained from $N$ by one rLST operation and $\beta^{r}\left(N^{\prime}\right)<\beta^{r}(N)=m$. The theorem now follows by induction.

By the above theorem, it follows that rLST operations induce a metric $d_{r \text { LST }}$ on the set of rooted level-1 networks on $X$ in which the distance $d_{r \mathrm{LST}}\left(N, N^{\prime}\right)$ between $N$ and $N^{\prime}$ is defined as the smallest number of rLST operations needed to convert $N$ to $N^{\prime}$. This is a new metric on rooted level-1 phylogenetic networks based on local operations, and it would be interesting to compare it with other known metrics on such networks (see, e.g. Cardona et al, 2011; Gambette and Huber, 2012; Huber and Moulton, 2013; Nakhleh, 2010, and the references therein). Finally, we remark here that it is possible to work out a formula to compute the size of the $d_{r \text { LST }}$ neighborhood of a rooted level-1 network via an approach similar to the proof of Theorem 3, but we shall not present this here as it is quite involved.

\section{Discussion}

In this paper, we have introduced some local operations on rooted and unrooted phylogenetic networks which generalize and extend the NNI operation on rooted and unrooted phylogenetic trees. We have shown that they have some attractive properties for unrooted level-1 networks, and that they can also be modified for use on rooted level-1 networks.

The local operations that we have defined can be applied to any unrooted phylogenetic network, independent of its level. It would be interesting to know whether or not they are sufficient to convert an arbitrary network into another one (also for rooted networks), or whether it is necessary to define additional operations. If new operations are required, we believe that reversibility will be a key component in their definition, and so it will probably be necessary to better understand whether or not excluding 3-cycle edges, links and chords will be sufficient to allow for reversible operations on general networks. Note that the operations that we have defined can potentially change the level of a network and so it would be necessary to adapt or introduce new operations if we wanted operations to preserve the level. More generally, it could also be of interest to understand how to define operations on special classes of networks which are somewhat easier to handle (e.g. tree-child networks (Cardona et al, 2009)), and to study spaces of such networks. Of course, the challenge will then be to ensure that we define operations that are guaranteed to produce networks that stay within any fixed class.

We should also mention that there are other operations that generalize NNI moves which are often also considered for phylogenetic trees, namely subtree prune and regraft $(S P R)$ and tree bisection and reconnection (TBR) operations (see e.g Allen and Steel, 2001). These are not local in nature as branches are cut in a tree and the resulting subtree can then be reinserted anywhere in the remaining graph. Probably SPR and TBR operations can be extended naturally to phylogenetic networks in general, but it will be much harder to control the behavior of the network that results from a 
single operation. To do this we may also need a concept extending agreement forests to networks (see e.g. Ding et al, 2011). Note that some non-local operations on rooted networks are defined in (Yu et al, 2014) that could be of interest in this context.

In general, there are several open problems concerning the network spaces that we have defined. For example, Gordon et al (2013) show that the spaces of phylogenetic trees induced by the NNI operation can be traversed by Hamiltonian paths and it would be interesting to see whether a similar result holds for the spaces of level-1 phylogenetic networks that we introduced (this is the case for $n=4$, following a straightforward check on Fig. 1). Also, it would be interesting to understand how the metric space of unrooted phylogenetic trees with the NNI metric is related to the space of unrooted level-1 networks with the LST metric, in particular to determine whether or not the tree space isometrically embeds into the network space.

Finally, using our operations, it should be possible to develop search algorithms which will allow users to systematically search through phylogenetic networks using scoring approaches such as parsimony and likelihood, as well as Bayesian approaches. Even so, this will also require some insights into the challenging problem on how to best score phylogenetic networks. Hopefully, understanding how scoring schemes operate on NNI neighborhoods of networks might give us some important clues to this problem.

Acknowledgements The authors thank the organizers of the workshop "Utilizing Genealogical Phylogenetic Networks in Evolutionary Biology: Touching the Data" at the Lorentz Center (the Netherlands) where the ideas for this paper were first discussed. We also thank the anonymous referee for very careful reading and helpful suggestions on the first version of this manuscript.

\section{References}

Allen B, Steel M (2001) Subtree transfer operations and their induced metrics on evolutionary trees. Annals of Combinatorics 5:1-15

Baroni M, Grünewald S, Moulton V, Semple C (2005) Bounding the number of hybridisation events for a consistent evolutionary history. Journal of mathematical biology 51(2):171-182

Billera LJ, Holmes SP, Vogtmann K (2001) Geometry of the space of phylogenetic trees. Advances in Applied Mathematics 27(4):733-767

Bordewich M, Semple C (2005) On the computational complexity of the rooted subtree prune and regraft distance. Annals of combinatorics 8(4):409_ 423

Cardona G, Llabres M, Rosselló F, Valiente G (2011) Comparison of galled trees. IEEE/ACM Transactions on Computational Biology and Bioinformatics (TCBB) 8(2):410-427

Chen T, Skiena SS (1996) Sorting with fixed-length reversals. Discrete Applied Mathematics 71(1):269-295

DasGupta B, He X, Jiang T, Li M, Tromp J, Zhang L (1997) On distances between phylogenetic trees. In: Proceedings of the eighth annual ACMSIAM symposium on Discrete algorithms, Society for Industrial and Applied Mathematics, pp 427-436

Day WH (1983) Properties of the nearest neighbor interchange metric for trees of small size. Journal of Theoretical Biology 101(2):275-288

Ding Y, Grünewald S, Humphries P (2011) On agreement forests. Journal of Combinatorial Theory, Series A 118(7):2059-2065

Gambette P, Huber K (2012) On encodings of phylogenetic networks of bounded level. Journal of Mathematical Biology 65(1):157-180

Gambette P, Berry V, Paul C (2009) The structure of level-k phylogenetic networks. In: Combinatorial Pattern Matching, Springer, pp 289-300

Gambette P, Berry V, Paul C (2012) Quartets and unrooted phylogenetic networks. Journal of bioinformatics and computational biology 10(04)

Gordon K, Ford E, John KS (2013) Hamiltonian walks of phylogenetic treespaces. IEEE/ACM Transactions on Computational Biology and Bioinformatics 10(4):1076-1079

Gusfield D (2014) ReCombinatorics: The Algorithmics of Ancestral Recombination Graphs and Explicit Phylogenetic Networks. MIT Press

Huber KT, Moulton V (2013) Encoding and constructing 1-nested phylogenetic networks with trinets. Algorithmica 66(3):714-738

Huber KT, van Iersel L, Kelk S, Suchecki R (2011) A practical algorithm for reconstructing level-1 phylogenetic networks. IEEE/ACM Transactions on Computational Biology and Bioinformatics (TCBB) 8(3):635-649 
Humphries P, Wu T (2013) On the neighborhoods of trees. IEEE/ACM Transactions on Computational Biology and Bioinformatics 10:721-728

Huson DH, Rupp R, Scornavacca C (2010) Phylogenetic Networks: Concepts, Algorithms and Applications. Cambridge University Press

Jansson J, Nguyen NB, Sung WK (2006) Algorithms for combining rooted triplets into a galled phylogenetic network. SIAM Journal on Computing 35(5):1098-1121

Jin G, Nakhleh L, Snir S, Tuller T (2006) Maximum likelihood of phylogenetic networks. Bioinformatics 22(21):2604-2611

Jin G, Nakhleh L, Snir S, Tuller T (2007) Inferring phylogenetic networks by the maximum parsimony criterion: a case study. Molecular Biology and Evolution 24(1):324-337

Keijsper J, Pendavingh R (2014) Reconstructing a phylogenetic level-1 network from quartets. Bulletin of Mathematical Biology 76:2517-2541

Lemey P, Salemi M, Vandamme AM (2009) The phylogenetic handbook: a practical approach to phylogenetic analysis and hypothesis testing. Cambridge University Press

Li M, Tromp J, Zhang L (1996) On the nearest neighbour interchange distance between evolutionary trees. Journal of Theoretical Biology 182(4):463467

Moore GW, Goodman M, Barnabas J (1973) An iterative approach from the standpoint of the additive hypothesis to the dendrogram problem posed by molecular data sets. Journal of Theoretical Biology 38(3):423-457

Moulton V, Wu T (2015) A parsimony-based metric for phylogenetic trees. Advances in Applied Mathematics 66:22-45

Nakhleh L (2010) A metric on the space of reduced phylogenetic networks. IEEE/ACM Transactions on Computational Biology and Bioinformatics (TCBB) 7(2):218-222

Radice R (2011) A Bayesian approach to phylogenetic networks. PhD thesis, University of Bath

Robinson DF (1971) Comparison of labeled trees with valency three. Journal of Combinatorial Theory, Series B 11(2):105-119

Rosselló F, Valiente G (2009) All that glisters is not galled. Mathematical biosciences 221(1):54-59

Semple C, Steel M (2003) Phylogenetics. Oxford University Press

Semple C, Steel M (2006) Unicyclic networks: compatibility and enumeration. IEEE/ACM Transactions on Computational Biology and Bioinformatics (TCBB) 3(1):84

Strimmer K, Moulton V (2000) Likelihood analysis of phylogenetic networks using directed graphical models. Molecular Biology and Evolution 17(6):875-881

Swofford DL, Olsen GJ, Waddell PJ, Hillis DM (1996) Phylogenetic inference. Sinauer Associates, Inc.

Yu Y, Dong J, Liu KJ, Nakhleh L (2014) Maximum likelihood inference of reticulate evolutionary histories. Proceedings of the National Academy of Sciences 111(46):16,448-16,453 